PACIFIC JOURNAL OF MATHEMATICS

Vol. 177, No. 1, 1997

\title{
GEODESIC FLOWS ON HYPERBOLIC ORBIFOLDS, AND UNIVERSAL ORBIFOLDS
}

\author{
María Teresa Lozano and José María Montesinos-Amilibia
}

The geodesic flow of a compact hyperbolic 2-orbifold is described. It is a flow in a 3-manifold which is Anosov out of a finite number of orbits. We study this class of flows (singular Anosov flows) and prove the existence of singular Anosov flows on every 3-manifold.

\section{Introduction.}

Anosov flows form a well-known class of flows. This class contains the geodesic flow of a hyperbolic surface $([\mathbf{1}],[6])$; the suspension flow of an Anosov diffeomorphism of the torus; and quotients of an Anosov flow by some flow-preserving group (algebraic flows). Methods have been developped to obtain Anosov flows by surgery ([10], [5], [8]), or by pasting two manifolds with Anosov flows together by Anosov diffeomorfism along boundary tori [15]. In all these examples the involved manifolds are closed and three-dimensional. However, not every 3-manifold admits an Anosov flow: for instance, no Anosov flow can exist in the sphere $S^{3}([\mathbf{1 6}])$.

It is natural to study geodesic flows of hyperbolic 2-orbifolds. They belong to a class of flows (Anosov flows with singular orbits), which we call VAnosov flows to convey the idea that the 2-fold covering of a V-Anosov flow branched over the singular set is an Anosov flow. It turns out that this class of flows can be placed in a more general class, (the class of singular Anosov flow) which are Anosov out of a finite number of closed orbits. An important reason to define this more general class is that the suspension flow of a generalized pseudo-Anosov diffeomorphism (in the sense of Laudenbach [7]) is a singular Anosov flow.

In this paper we introduce these concepts and show the following:

Section 1: Definition of singular Anosov and V-Anosov flows.

Section 2: Characterization of transitive Anosov flow in 3-manifolds (correcting a result of Fried [8]).

Section 3: Description of the geodesic flows of hyperbolic orbifolds. 
Section 4: Study of the singular links of geodesic flows of hyperbolic orbifolds (when the underlying 3-manifold is $S^{3}$ ). Constructions of VAnosov flows in $S^{3}$ by other procedures. As an application we give a result about links in $S^{3}$ (Corollary 4.2.2).

Section 5: Proof that every 3-manifold has a singular Anosov flow (with some extra-control of the flow). This is done after proving the existence of links $L$ in $S^{3}$ both universal and singular set of $\mathrm{V}$-Anosov flows. (A link is universal if every 3 -manifold is covering of $S^{3}$ branched over $L$.)

Known concepts of flows not defined in this paper can be found in [22]. References for concepts related with links are [23] and [4]. For a 3-manifold we usually mean a closed, orientable 3 -manidold, but 2-orbifolds do not need to be orientable; on the contrary, the interesting examples arise from the geodesic flows of non-orientable hyperbolic orbifolds.

\section{$\S 1$. Singular Anosov flows.}

The concept of pseudo-Anosov diffeomorphism of a surface was introduced by Thurston in [24], for the classification of diffeomorphisms of surfaces. It was soon generalized by Laudenbach [7] by allowing a new kind of singular points (1-prong singularities, see Definition 1.1 below) which is basic in our work.

Definition 1.1 [7, p. 243]. A generalized pseudo-Anosov diffeomorphism is a diffeomorphism $f: S \rightarrow S$ of a surface $S$ having two mutually transverse measured foliations, $\left(\mathbf{F}^{s}, \mu^{s}\right),\left(\mathbf{F}^{u}, \mu^{u}\right)$ and a real number $\lambda>1$ satisfying $f\left(\mathbf{F}^{s}, \mu^{s}\right)=\left(\mathbf{F}^{s}, \lambda^{-1} \mu^{s}\right)$ and $f\left(\mathbf{F}^{u}, \mu^{u}\right)=\left(\mathbf{F}^{u}, \lambda \mu^{u}\right)$. The foliations $\mathbf{F}^{s}, \mathbf{F}^{u}$ mitght have a finite number of $k$-prong singularities $(k=1$, or $k \geq 3)$ which form the singular set of $f$. (Fig. 1.1.)

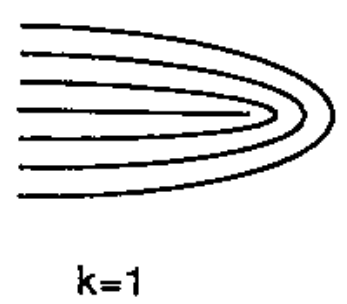

$k=1$

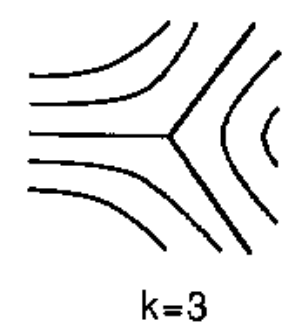

Figure 1.1.

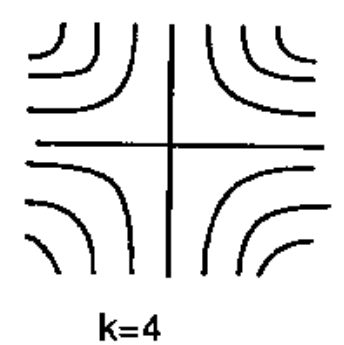

$\mathrm{k}=4$

Note that:

1) A point in the singular set of $\mathrm{f}$ is a $k$-prong of both foliations $\mathbf{F}^{s}, \mathbf{F}^{u}$ since they are transverse. Such a point will be called a $k$-singular point of $f$. 
2) The restriction of $\mathrm{f}$ to $S \backslash P$, where $P$ is the set of 1-prong singularities, is a pseudo-Anosov diffeomorphism. (See [24], [21], [7].)

3) Let $S^{\prime}$ be a double cover of $S$ branched over a finite set of points $P^{\prime}$ including $P$. A lifting $f^{\prime}$ of $f$ to $S^{\prime}$ is a pseudo-Anosov diffeomorphism, because the singularities of $f^{\prime}$ are $k$-prong, $k \geq 3$.

Definition 1.1.2. A flow $\varphi$ on a closed 3-manifold $M$ is a singular Anosov flow if, first, there exists a union $\Gamma$ of a finite number of periodic orbits $\gamma_{1}, \ldots \gamma_{n}$, such that the restriction of $\varphi$ to $M \backslash \Gamma$ is an Anosov flow, and, second, a Poincaré map for a transversal local section to $\gamma_{i}$ in a point $p_{i}$, $i=1, \ldots, n$, is a generalized pseudo-Anosov diffeomorphism whose singular set consists of the $k_{i}$-prong singular point $p_{i}$.

The orbits of $\Gamma$ form the singular set of the flow. We will say that $\gamma_{i}$ is a $k_{i}$-singular orbit of $\varphi$. A 1-singular orbit will be called a thorn.

A $V$-Anosov flow is a singular Anosov flow, whose singular set consists only of thorns.

The concept of singular Anosov flow is important in relation with the construction of all transitive Anosov flows in 3-manifolds (see §2). We will see that singular Anosov flows appear in a natural way as the geodesic flows of 2-hyperbolic orbifolds (see $\S 3$ ). It is shown in $\S 5$ that every closed oriented 3-manifold admits singular Anosov flows.

Note that the suspension construction applied to a generalized pseudoAnosov diffeomorphisms yields a singular Anosov flow. Indeed, let $S$ be a closed surface, and let $f: S \rightarrow S$ be a generalized pseudo-Anosov diffeomorphism. Consider the 3-manifold $M=S \times[0,1] /(x, 1) \sim(f(x), 0)$. The suspension flow $\varphi$ on $M$ is defined by $\varphi((x, s), t)=\left(f^{[t]}(x), s+t-[t+s]\right)$, where $(x, s)$ is a point of $M$ and [*] denotes the integer part of $*$. Then, $\varphi$ is a singular Anosov flow.

\section{§2. Transitive Anosov flows in 3-manifolds.}

\subsection{Dehn surgery on periodic orbits of singular Anosov flows.}

Construction 2.1.1. Let $\varphi$ be a singular Anosov flow in a 3-manifold $M^{3}$, and let $\gamma$ be a periodic orbit of $\varphi$. We describe now, a process that (while changing the manifold by Dehn surgery along $\gamma$ ) it modifies the flow in a neighborhood of $\gamma$. (Compare [10], [8], [5].) Note that if this process is applied to an Anosov flow whose stable foliations are infinitely smooth, the stable foliations of the new flow might not be infinitely smooth any more $([\mathbf{9}])$.

The process is done in two steps: 
A. Blowing up. A tubular neighborhood of $\gamma, N(\gamma)$, is homeomorphic to $D^{2} \times S^{1}$, where $\gamma$ corresponds to $(0,0) \times S^{1}$. The disk $D^{2}$ can be considered as the quotient of $S^{1} \times[0,1]$ by the relation $(\theta, 0) \sim\left(\theta^{\prime}, 0\right)$. In this step we forget the relation $\sim$ in $D^{2} \times S_{1} \cong N(\gamma)$, so that the manifold $M$ is transformed in $M^{*}$, where the curve $\gamma$ has been replaced by a boundary torus $T$. In $M^{*}$ there exists a flow $\varphi^{*}$ such that $\left.\left.\varphi\right|_{M \backslash \gamma} \equiv \varphi^{*}\right|_{M \backslash T}$, and the restriction of $\varphi^{*}$ to $T$ is a Morse Smale flow with atractors and repellers alternating as in Fig. 2.1. Let $(\mu, \lambda)$ be a homology basis in $T$, where $\mu \sim \partial D^{2}$ and $\lambda \sim \gamma$ in $N(\gamma)$. The number of intersection points of $\mu$ with the set of atractors is either 2 , if $\gamma$ is an ordinary orbit, or $k$, if $\gamma$ is a $k$-singular orbit. (See Fig. 2.1.)

Note that in the case of an ordinary orbit, for instance, the number of attractors of $\left.\varphi^{*}\right|_{T}$ can be one or two, according as if the component of the intersection of $N(\gamma)$ with the stable leaf of $\gamma$ in $\varphi, W_{\gamma}^{s}[22$, p. 98], is a Möebius band or an annulus. (See Fig. 2.1.a).) The Möebius band case was not considered neither by Christy [5] nor Fried [8].
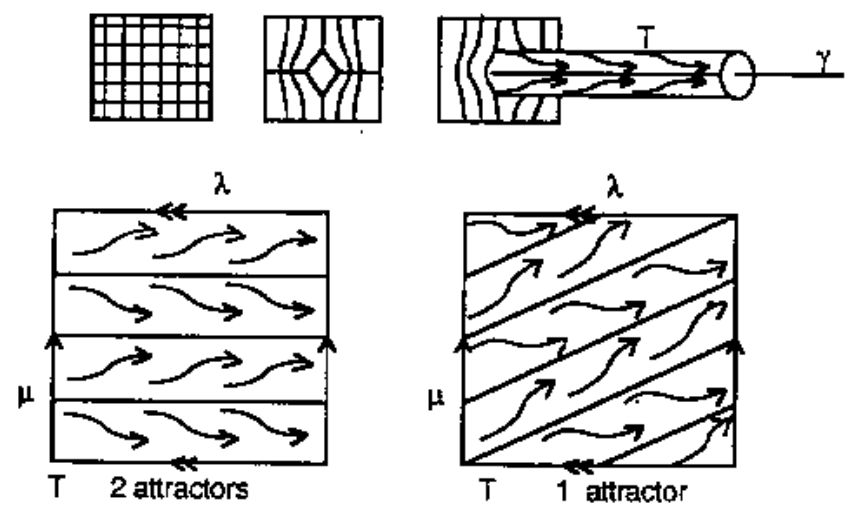

Figure 2.1. a) Ordinary orbit
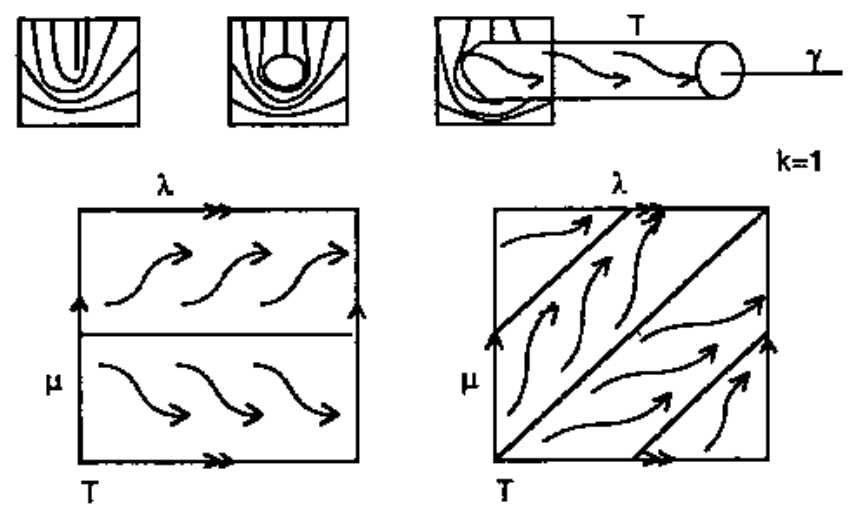

Figure 2.1. b) 1-singular orbit $(k=1)$ 


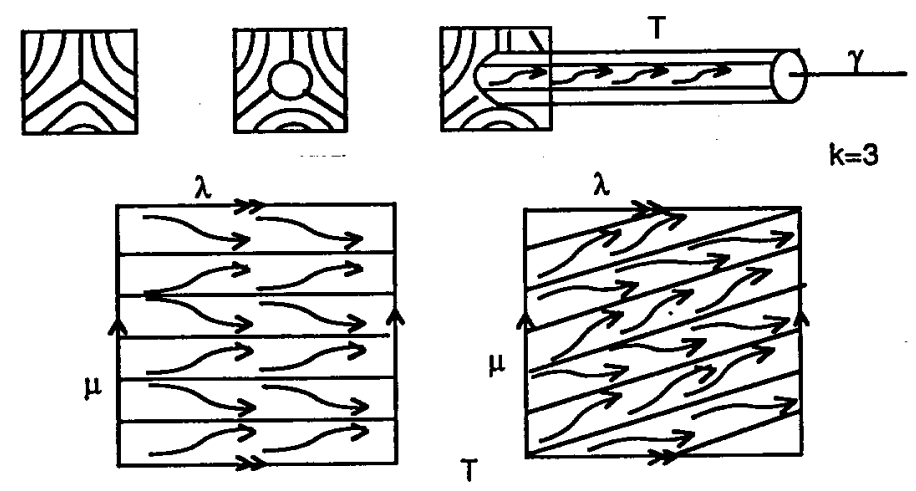

Figure 2.1. c) 3 -singular orbit $(k=3)$

B. Blowing down. Consider the torus $T$ fibred by curves $Q=p \lambda+q \mu$, where $p$ and $q$ are relative prime integers, and collapse each fiber to a point. Let $\gamma^{\prime}$ be the set of these points. The resulting manifold $M^{\prime}=M(q / p)$ is homeomorphic to the manifold obtained from $M$ by Dehn surgery along $\gamma$, with coefficient $r=q / p$. The flow $\varphi^{*}$ induces a flow $\varphi^{\prime}$ on $M^{\prime}$ such that $\left.\left.\left.\varphi^{\prime}\right|_{M^{\prime} \backslash \gamma^{\prime}} \equiv \varphi^{*}\right|_{M \backslash T} \equiv \varphi\right|_{M \backslash \gamma}$. Let $\left(p_{0}, q_{0}\right)$ be the couple (well defined up to change of sign) of relatively prime integers such that $Q_{0}=p_{0} \lambda+q_{0} \mu$ is homologous to an atractor of the Morse Smale flow in $T$, (thus $Q_{0}$ is homologous to every attractor and repeller of the Morse Smale flow in $T$ ). Then the flow $\varphi^{\prime}$ in $M\left(p_{0}, q_{0}\right)$ has fixed points, namely the images in $\gamma^{\prime}$ of the attractors and repellers of $T$. Only in this case $\varphi^{\prime}$ is not a singular Anosov flow in $M^{\prime}$. For $(p, q) \neq\left(p_{0}, q_{0}\right), \gamma^{\prime}$ is a periodic orbit of $\varphi^{\prime}$, ordinary or singular, depending on the choice of $(p, q)$ and $\left.\varphi^{*}\right|_{T}$, and $\varphi^{\prime}$ is again a singular Anosov flow. To see this, just consider an appropriate local section to $\gamma^{\prime}$.

\subsection{Construction of Anosov flows.}

Construction 2.2.1. Let $S$ be a closed surface, and let $f: S \rightarrow S$ be a generalized pseudo-Anosov diffeomorphism.

Let $p \in S$ be a $k$-singular point of $f, k=1$ or $k \geq 3$. The smallest period of $p$ is denoted by $m(p)$. This is the smallest natural number $m$ such that $f^{m}(p)=p$. Let $j=j(p)$ be the smallest integer such that $f^{m j}$ carries each prong at $\mathrm{p}$ into itself (each singular leaf going to itself). Clearly, $j \mid k$. We assume that the diffeomorphism $f$ is such that $\frac{k}{j} \in\{1,2\}$ for every $k$-singular point $p$. This hypothesis is necessary for the following construction.

Let $F$ be a finite set of points of $S$ invariant for $f$ and containing the singular set of $f$. We consider the 3-manifold $M=S \times[0,1] /(x, 1) \sim(f(x), 0)$ 
endowed with the suspension flow $\varphi$, which is a singular Anosov flow (see $\S 1)$. Let $\left(M^{*}, \varphi^{*}\right)$ be obtained from $(M, \varphi)$ by blowing up the set of orbits $F \times[0,1] /(x, 1) \sim(f(x), 0)$ (see $\S 2.1)$. The flow $\varphi^{*}$ restricted to the boundary of $M^{*}$ is a Morse-Smale flow. We choose a homology basis $(\lambda, \mu)$ for each boundary component, and we call $H$ the homology class represented by an attractor of the flow (and therefore by any attractor or repeller). For a suitable $\lambda$, the coordinates of $H$, which depend on $k$ and $j$, are the following:

$$
\left\{\begin{array}{lll}
H=\frac{k}{2} \lambda-\mu & j=\frac{k}{2}, & 2 \text { attractors and } 2 \text { repellers } \\
H=k \lambda-\mu & j=k, & 1 \text { attractor and } 1 \text { repeller. }
\end{array}\right.
$$

Fig. 2.2 represents the particular cases $k=1,2$.

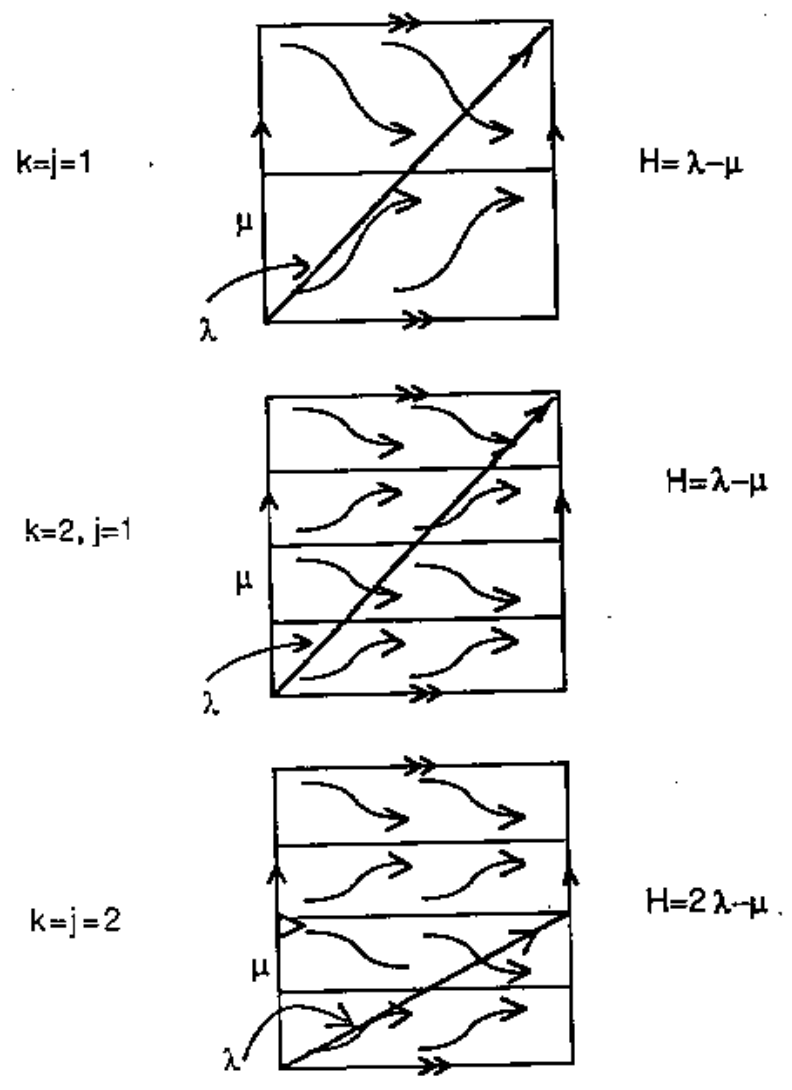

Figure 2.2.

We blow down every boundary component of $M^{*}$ choosing a couple $(p, q)$ such that the new orbit is an ordinary orbit of the flow $\varphi^{\prime}$ in the resulting manifold $M^{\prime}$. The couple $(p, q)$ is obtained by requiring that the number of 
intersection points of $Q=p \lambda+q \mu$ with the set of attractors and repellers is 4. The possibilities are the following:

$$
\begin{array}{rll}
\text { for } k=1 & p \text { odd, } & q=2 \\
\text { for } k \text { even and } j=\frac{k}{2} & k q+2 p=2, & \text { g.c.d }(p, q)=1 \\
\text { for any } k \text { and } j=k & k q+p=2, & \text { g.c.d }(p, q)=1 .
\end{array}
$$

The flow $\varphi^{\prime}$ in the new closed 3-manifold $M^{\prime}$ is an Anosov flow because it is a singular Anosov flow with empty singular set.

Theorem 2.2.2. Let $\varphi$ be a transitive Anosov flow on a closed 3-manifold $M$. If $\varphi$ has a surface of section then $\varphi$ is topologically conjugate to one obtained by the above construction.

Proof. Let $S$ be a surface of section for the flow $\varphi$ and let $\Gamma$ be the set of the $n$ periodic orbits constituting the boundary of $S$. Let $f$ be the Poincare map of $S \backslash \partial S$. Let $M^{*}$ be the result of blowing up the orbits contained in $\Gamma$. There is a unique compactification, $\hat{S}$, of $S \backslash \partial S$ with a point $P_{i}$ on each end (Freudenthal compactification). On each boundary component $T_{i}$ of $M^{*}$, $(i=1, \ldots, n)$, consider the well defined curve $Q_{i}=T_{i} \cap S$. Let $M_{1}$ be the manifold obtained by blowing down every boundary torus $T_{i}$ of $M^{*}$, using $Q_{i}$ as fiber, $(i=1, \ldots, n)$. Let $\varphi_{1}$ be the induced flow on $M_{1}$. Note that $M_{1}$ contains the closed surface $\hat{S}$ as a global section of the flow $\varphi_{1}$. Then, the manifold $M_{1}$ is $\hat{S} \times[0,1] /(x, 1) \sim\left(f_{1}(x), 0\right)$, where the extension $f_{1}$ of $f$ to $\hat{S}$ is a generalized pseudo-Anosov diffeomorphism. It follows that the suspension flow $\psi$ in $M_{1}$ is topologically equivalent to $\varphi_{1}$. Note that this process might create 1-prong singularities in $f_{1}$. (See Note 1 below.)

Now, we prove that the flow $\varphi$ on $M$ is topologically conjugate to one obtained by Construction 2.2.1: We start from the mapping torus $M_{1}$ of $f_{1}$, and the corresponding suspension flow $\psi$. Then, we perform Construction 2.2.1 by reversing the precedent process, as follows:

a) Blow up the periodic orbits of $\psi$ passing through the points $P_{i},(i=$ $1, \ldots, n)$, of $\hat{S}$, to obtain $M^{*}$.

b) Blow down the boundary components of $M^{*}$ to obtain $M$. The induced flow in $M$ is topologically conjugate to $\varphi$.

Corollary 2.2.3. Any transitive Anosov flow on a closed 3-manifold is topologically conjugate to one obtained by Construction 2.2.2.

Proof. Theorem 2 of [8] establishes that every transitive Anosov flow on a closed 3-manifold has a surface of section. Then, apply the precedent Theorem 2.2.1. 
Notes. 1). Fig. 2.3 shows an example of a boundary torus Tof a manifold $M^{*}$ obtained by blowing up a periodic orbit which is a boundary component of a surface of section of a flow $\varphi$ in a 3-manifold $M$. The Morse-Smale flow in $T$ has an attractor and a repeller. The line $R$ is the intersection of $T$ and $S$. It is clear that the process of blowing down defined by $R$ produces a singular Anosov flow with a thorn. Concrete examples of this situation can be constructed from the flows of section 4. (See 4.4.2 ii).) This case was no considered by Fried [8].

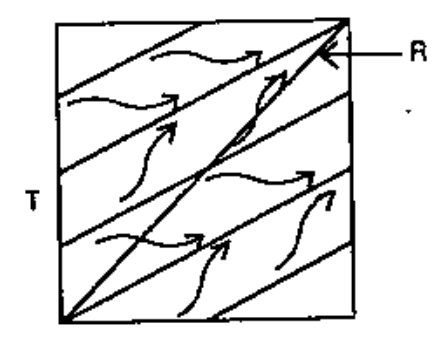

Figure 2.3.

2). Theorem 1 of [8] would be correct if it were always possible to find a surface of section such that the flow $\varphi_{1}$ in the manifold $M_{1}$ has no thorns. This still is an open question.

\section{$\S 3 . S T(Q)$ and its geodesic flow.}

3.1. Geodesic flow of compact hyperbolic 2-orbifolds. Let $Q$ be a compact hyperbolic 2-orbifold. There exists a subgroup $\Gamma$ of the group Iso $\left(\mathbb{H}^{2}\right)$ of isometries of the hyperbolic plane $\mathbb{H}^{2}$ such that $\Gamma$ acts on $\mathbb{H}^{2}$ on the left as a properly discontinuous transformation group, and the quotient $\Gamma \backslash \mathbb{H}^{2}$ is $Q$. The total space $S T(Q)$ of the spherical tangent bundle of $Q$, is a 3 -manifold, which is the quotient of the total space $S T\left(\mathbb{H}^{2}\right)$ of the spherical tangent bundle of $\mathbb{H}^{2}$ by the action of $\Gamma$ on the tangent space of $\mathbb{H}^{2}$ on the left (i.e. $S T(Q)=\Gamma \backslash S T\left(\mathbb{H}^{2}\right)$ ). (See, for instance [19, p. 91].)

The geodesic flow $\varphi$ for $\mathbb{H}^{2}$ is defined on $S T\left(\mathbb{H}^{2}\right)$ by

$$
\begin{aligned}
\varphi: S T\left(\mathbb{H}^{2}\right) \times \mathbb{R} & \rightarrow S T\left(\mathbb{H}^{2}\right) \\
((x, v), t) & \rightarrow\left(x^{\prime}, v^{\prime}\right) .
\end{aligned}
$$

Here $x^{\prime}=\gamma_{x}^{v}(t)$, where $\gamma_{x}^{v}$ is the geodesic defined by $(x, v)$, and $v^{\prime}$ is the tangent vector to $\gamma_{x}^{v}$ at $x^{\prime}$. Clearly, the orbit of a point $(x, v)$ consists of the tangent vectors to the geodesic defined by $(x, v)$.

The isometries of $\Gamma$ map geodesics of $\mathbb{H}^{2}$ onto geodesics, and therefore the geodesic flow $\varphi$ is $\Gamma$-equivariant. The quotient of the geodesic flow $\varphi$ for $\mathbb{H}^{2}$ 
by the action of $\Gamma$ is, by definition, the geodesic flow for the 2-orbifold $Q$. It is a flow $\psi$ on $S T(Q)$. We are interested in the study of this flow.

First, we recall the properties of the geodesic flow for $\mathbb{H}^{2}$. It is obviously a flow in the 3-manifold $S T\left(\mathbb{H}^{2}\right)$. This manifold can be identified with $\mathbb{H}^{2} \times S^{1}$ by the diffeomorphism

$$
\begin{aligned}
S T\left(\mathbb{H}^{2}\right) & \rightarrow \mathbb{H}^{2} \times S^{1} \\
(x, v) & \rightarrow(x, \theta)
\end{aligned}
$$

where $\theta \in S^{1}$ is the $\alpha$-limit point of $(x, v)$ (i.e. $\theta=\lim _{t \rightarrow \infty} \gamma_{x}^{v}(t)$, where $\gamma_{x}^{v}$ is the geodesic defined by $(x, v)$ ). (See Fig. 3.1.) The geodesic flow defines a foliation on $S T\left(\mathbb{H}^{2}\right)$, such that each orbit of the flow is a leave of the foliation. Then, the leaves of the foliation are diffeomorphic to $\mathbb{R}$, and each $\mathbb{H}^{2} \times\{\theta\}$ is foliated by all the geodesics with $\theta$ as $\alpha$-limit point.

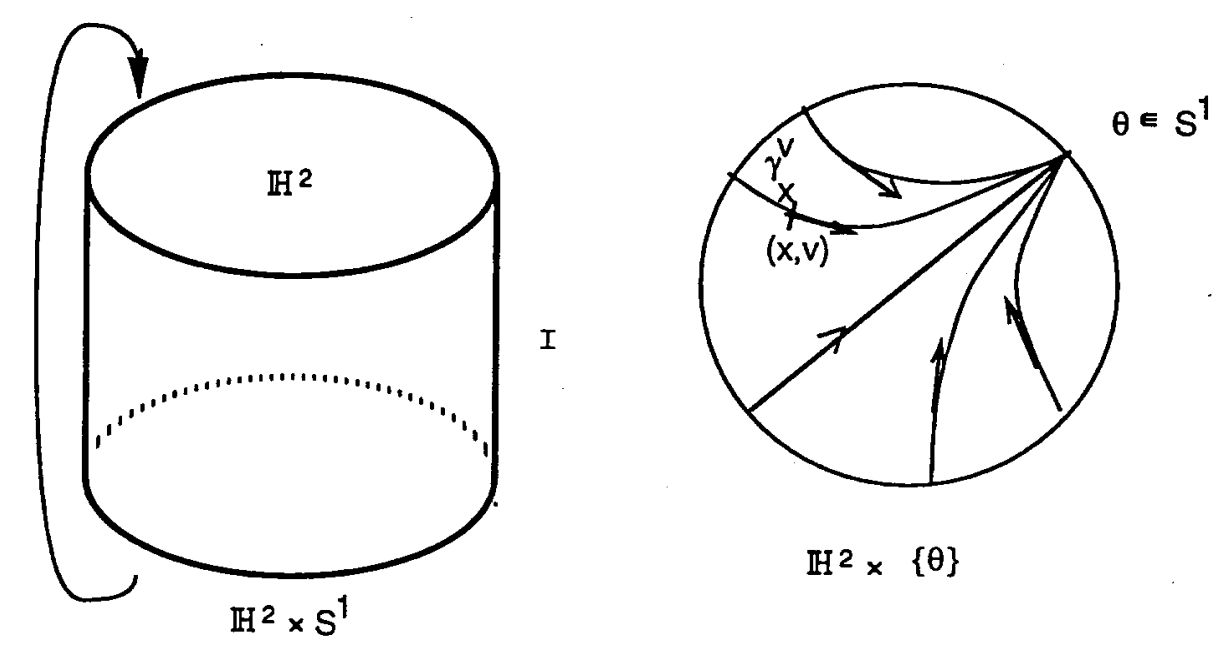

Figure 3.1.

The geodesic flow $\varphi$ on $S T\left(\mathbb{H}^{2}\right)$ is globally hyperbolic, which means that: (i) The unstable submanifold $W_{(x, v)}^{\varphi u}$ through the point $(x, v)$ consists, by definition, of the tangent vectors to the geodesics $\gamma$ with $\theta=\lim _{t \rightarrow-\infty} \gamma_{x}^{v}(t)$ as $\omega$-limit point (i.e. $\theta=\lim _{t \rightarrow-\infty} \gamma(t)$ ). (See Fig. 3.2 a).)

(ii) The stable submanifold $W_{(x, v)}^{\varphi s}$ through the point $(x, v)$ consists, by definition, of the tangent vectors to the geodesics $\gamma^{\prime}$ with $\theta=\lim _{t \rightarrow+\infty} \gamma_{x}^{v}(t)$ as $\alpha$-limit point (i.e. $\theta=\lim _{t \rightarrow+\infty} \gamma^{\prime}(t)$ ). (See Fig. 3.2 b).)

(iii) The unstable and stable manifolds $W_{(x, v)}^{\varphi u}$ and $W_{(x, v)}^{\varphi s}$ intersect transversally along to the tangent vectors of the geodesic $\gamma_{x}^{v}$, as it is shown in Fig. $3.2 \mathrm{c})$, in a neighborhood of $(x, v)$. 
a)
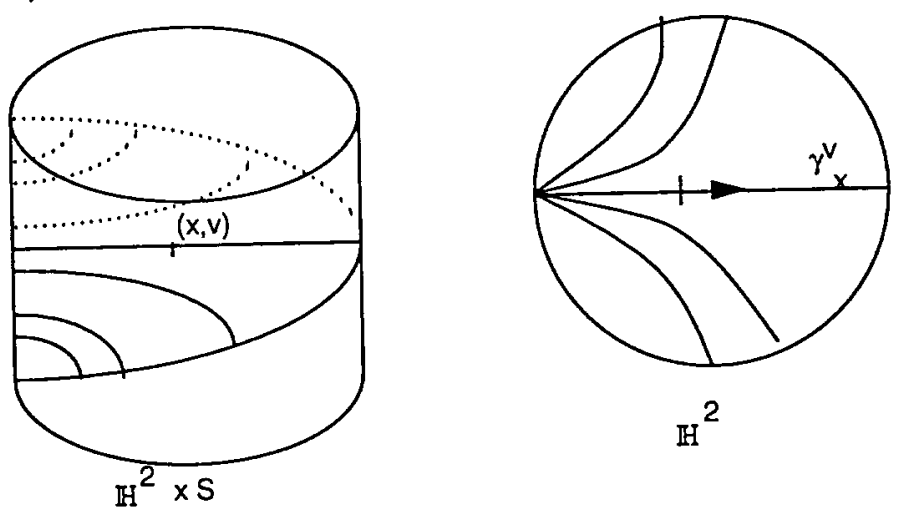

b)
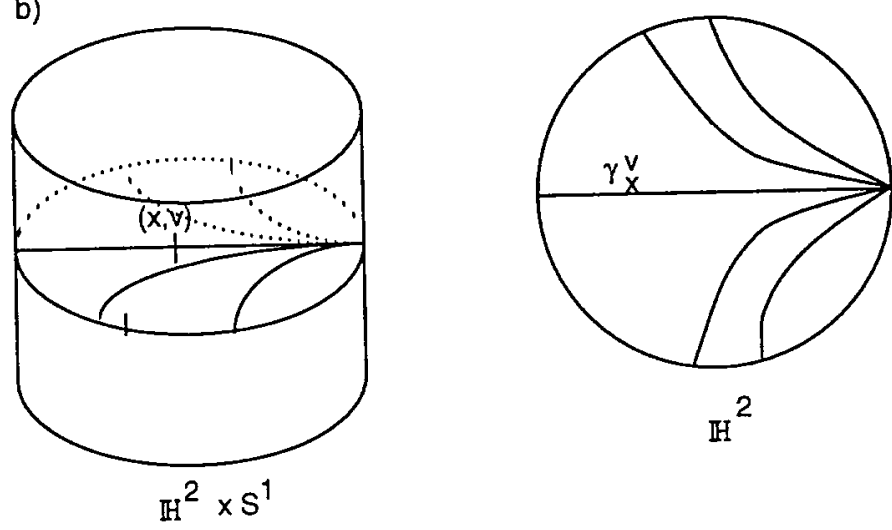

c)

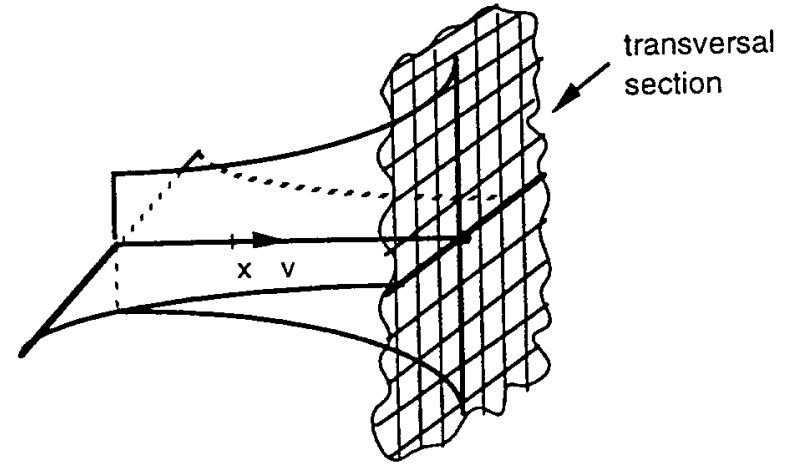

Figure 3.2.

Let $g$ be an isometry of $\mathbb{H}^{2}$. The action of $g$ on $S T\left(\mathbb{H}^{2}\right)$ is given by 
$g(x, v)=(g(x), d g(v))$. The image of $\left(\gamma_{x}^{v}(t), v^{\prime}\right) \subset \mathbb{H}^{2} \times\{\theta\}$ by $g$ is

$$
\left(\gamma_{g(x)}^{d g(v)}(t), v^{\prime \prime}\right) \subset \mathbb{H}^{2} \times\left\{\theta^{\prime}\right\}
$$

where $v^{\prime}$ is the tangent vector to $\gamma_{x}^{v}$ at $\gamma_{x}^{v}(t)$, and $v^{\prime \prime}$ is the tangent vector to $\gamma_{g(x)}^{d g(v)}$ at $\gamma_{g(x)}^{d g(v)}(t)$. Note that $g$ acts on $\partial \mathbb{H}^{2}$ sending $\theta$ to $\theta^{\prime}$. (Fig. 3.3.)

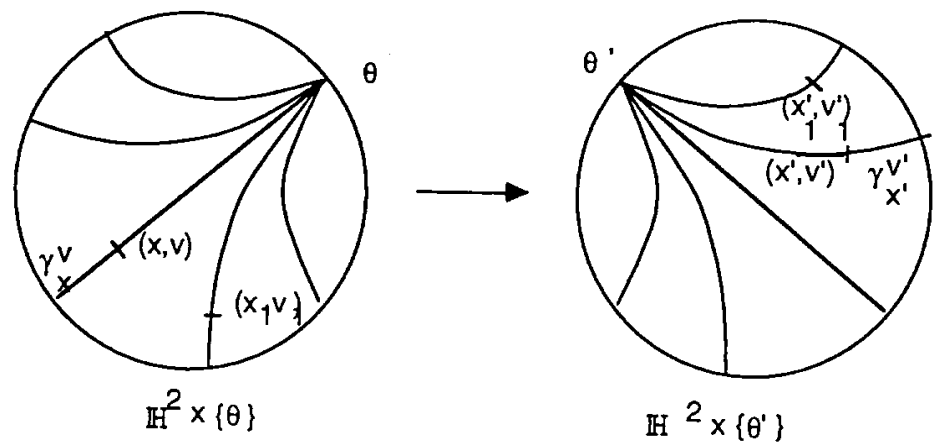

Figure 3.3.

The isometries of $\mathbb{H}^{2}$ acting with fixed points are reflections and $\frac{2 \pi}{n}$ rotations, $n \in \mathbb{Z}$. We now analyze its action on $S T\left(\mathbb{H}^{2}\right)$ and the effect on the projected flow $\psi$.

Lemma 3.1.1. Let $g$ be a reflection. Then the geodesic flow $\psi$ in $g \backslash \mathbb{H}^{2}$, is a $V$-Anosov flow. The singular set consists of two thorns.

Proof. Let $g$ be a reflection on a geodesic $\gamma$ of $\mathbb{H}^{2}$. The fix points of $g$ on $\mathbb{H}^{2}$ are the points of $\gamma$. Let $\bar{\gamma}_{1}$ be the geodesic $\gamma$ endowed with one of the two possible orientations, and let $\bar{\gamma}_{2}$ be the geodesic $\gamma$ endowed with the other possible orientation. The set $A_{i} \subset S T\left(\mathbb{H}^{2}\right)$ of tangent vectors to $\bar{\gamma}_{i}$ is the copy of $\gamma$ contained in $\mathbb{H}^{2} \times\left\{\theta_{i}\right\}, i=1,2$, where $\theta_{1}$ and $\theta_{2}$ are the $\alpha$-limit point and the $\omega$-limit point, respectively, of the geodesic $\bar{\gamma}_{1}$.

Next we prove that the set $F$ of fixed points of $g$ on $S T\left(\mathbb{H}^{2}\right)$ is $A_{1} \cup A_{2}$ : The point $(x, v)$ is an element of $F$, if and only if $(x, v)=g(x, v)=(g(x), d g(v))$. This equality holds if and only if $x \in \gamma$ and $v$ is a tangent vector to $\gamma$, which is equivalent to $(x, v) \in A_{1} \cup A_{2}$.

The natural projection $\pi: S T\left(\mathbb{H}^{2}\right) \longrightarrow g \backslash S T\left(\mathbb{H}^{2}\right)$ is a covering, branched over $\pi\left(A_{1} \cup A_{2}\right)$. Therefore the restriction of $\pi$ to $S T\left(\mathbb{H}^{2}\right)-\left(A_{1} \cup A_{2}\right)$ is a local diffeomorphism and the projected flow on $\left(g \backslash S T\left(\mathbb{H}^{2}\right)\right)-\pi\left(A_{1} \cup A_{2}\right)$ has the same local properties that $\varphi$, i.e. it is hyperbolic. The set $A_{i}, i=1,2$, which is an orbit of $\varphi$, projets onto the orbit $\pi\left(A_{i}\right)$ of the projected flow 
$\psi$. Let $N_{i}$ be the neighborhood of $A_{i}$ in $S T\left(\mathbb{H}^{2}\right)$ formed by the tangent vectors to geodesics with $\theta_{i} \pm \varepsilon$ as $\alpha$-limit point and with base point in a neighborhood of $\gamma$ invariant by the action of $g$ on $\mathbb{H}^{2} . N_{i}$ is invariant by the action of $g$ on $S T\left(\mathbb{H}^{2}\right)$. Actually, the action of $g$ on $N_{i}$ is a $\pi$-rotation around $A_{i}$. Figure 3.4 shows the neighborhood $N_{i}$ and the action on a transversal section to $A_{i}$. Then $\pi\left(A_{i}\right)$ is not hyperbolic in the projected flow $\psi$, but it is a thorn. We have prove that $\psi$ is a $V$-Anosov flow with two thorns, $\pi\left(A_{1}\right)$ and $\pi\left(A_{2}\right)$.
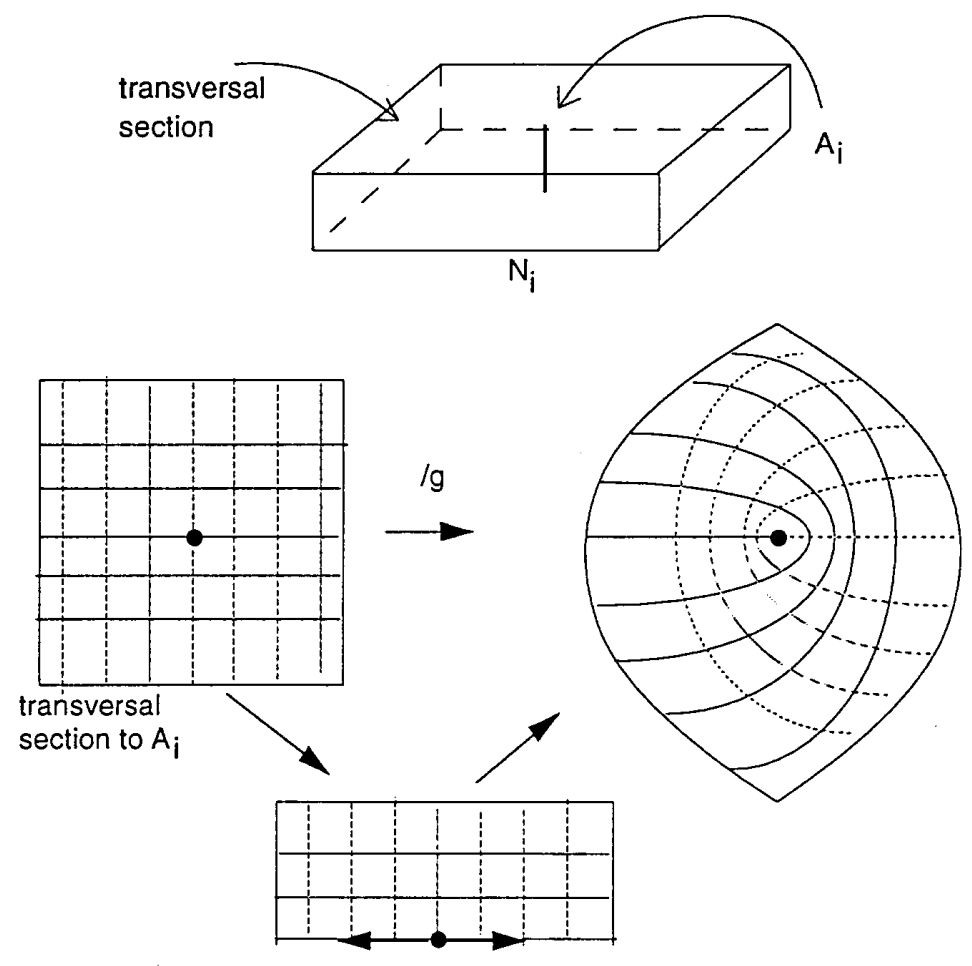

Figure 3.4.

Lemma 3.1.2. If $g$ is a $\frac{2 \pi}{n}$-rotation around a point $P$ or an isometry without fixed points, then the geodesic flow $\psi$ in $g \backslash \mathbb{H}^{2}$, is a singular Anosov flow with empty singular set.

Proof. Let $g$ be a $\frac{2 \pi}{n}$ - rotation around a point $P$ of $\mathbb{H}^{2}$ or an isometry without fixed points. Then $g$ acts on $S T\left(\mathbb{H}^{2}\right)$ without fixed points, therefore the natural projection $\pi: S T\left(\mathbb{H}^{2}\right) \longrightarrow g \backslash S T\left(\mathbb{H}^{2}\right)$ is a local diffeomorphism and thus the projected flow on $g \backslash S T\left(\mathbb{H}^{2}\right)$ is globally hyperbolic. 
Theorem 3.1.3. The geodesic flow of a compact hyperbolic 2-orbifold $Q$ is a $V$-Anosov flow on the closed 3-manifold $S T(Q)$.

Proof. Let $\Gamma$ be the subgroup of isometries of the hyperbolic plane $\mathbb{H}^{2}$, such that $Q=\Gamma \backslash \mathbb{H}^{2}$. The group $\Gamma$ is finitely generated because $Q$ is compact. Then there exists a fundamental domain $D$ for the action of $\Gamma$ on the plane $\mathbb{H}^{2}$ which is a polygon with a finite number of edges. Therefore $S T(Q)$ is obtained from $D \times S^{1}$ by identifyng its faces by the action of a finite number of elements of $\Gamma$. It follows from Lemma 3.1.1 and Lemma 3.1.2 that the geodesic flow for $Q$ on the manifold $S T(Q)$ is a $V$-Anosov flow.

Note that if a discrete group of isometries $\Gamma$ acts freely on $\mathbb{H}^{2}$, it acts also freely on $S T\left(\mathbb{H}^{2}\right)$. In this case $Q=\Gamma \backslash \mathbb{H}^{2}$ is a non singular 2-orbifold. In addition, if $\Gamma$ is cocompact, $\Gamma \backslash \mathbb{H}^{2}$ is a compact surface $S$ and the geodesic flow $\psi$ for $S$ is an Anosov flow [1]:

1) It is globally hyperbolic.

2) $\overline{\mathrm{Per}}=S T(S)$, where Per is the set of periodic orbits.

3.2. Seifert fibration on $S T(Q)$. The manifold $S T(Q)$ has the structure of a Seifert fibred orbifold which is the quotient of the trivial $S^{1}$-fibred bundle structure on $S T\left(\mathbb{H}^{2}\right)=\mathbb{H}^{2} \times S^{1}$. The manifold $S T(Q)$ has a decomposition into circles and compact intervals, corresponding to the fibers of the tangent bundle, with base the 2-orbifold $Q$. We will calculate the signature of this structure of $S T(Q)$ in the classification given by Bonahon-Siebenmann [3]. This signature generalizes the signature of a Seifert manifold to Seifert fibred orbifolds.

The signature consists of:

(i) A slope $\beta / \alpha$ (we write also $(\alpha, \beta)$ ) associated to each cone point with isotropy group cyclic of order $\alpha$, and to each corner point with isotropy group dihedral of order $2 \alpha$.

(ii) A parity $\varepsilon \in \mathbb{Z}_{2}$ associated to each mirror cycle.

(iii) The Euler class $e \in \frac{1}{2} \mathbb{Z}$.

Let $\mathrm{P}$ be a cone point whose isotropy group is the cyclic group $C_{\alpha}$ of order $\alpha$. Then $(\alpha, \beta)$ defines the exceptional fiber over $P$. To compute this slope we analyze the fibration in a neighborhood of the exceptional fiber. Let $h$ be the hyperbolic $\frac{2 \pi}{\alpha}$-rotation around $P$, where $\alpha \in \mathbb{N}$, and let $D^{2} \times S^{1}$ be a neighborhood of $P \times S^{1}$ in $S T\left(\mathbb{H}^{2}\right)$. The action of $h$ on $D^{2} \times S^{1}$ is given by $(h, d h)$.

$$
\begin{aligned}
D^{2} \times S^{1} & \longrightarrow D^{2} \times S^{1} \\
\left(\left(r, \theta_{1}\right), \theta_{2}\right) & \longrightarrow\left(\left(r, \theta_{1}+\frac{2 \pi}{\alpha}\right), \theta_{2}+\frac{2 \pi}{\alpha}\right) .
\end{aligned}
$$


It rotates $D^{2}$ and $S^{1}$ an angle $\frac{2 \pi}{\alpha}$. Thus $\beta=\alpha-1$ because the angle
$\frac{2 \pi}{\alpha}$ is equal to $-\frac{2 \pi(\alpha-1)}{\alpha}=-\frac{2 \pi \beta}{\alpha}$.
Let $\mathrm{P}$ be a corner point whose isotropy group is the dihedral group $\Delta_{2 \alpha}$ of order $2 \alpha$. Then $(\alpha, \beta)$ is also $(\alpha, \alpha-1)$, because the generator of $C_{\alpha} \leq \Delta_{2 \alpha}$ produces a $\frac{2 \pi}{\alpha}$-rotation around $P$, and each element of $\Delta_{2 \alpha} \backslash C_{\alpha}$ acts by reflection on $D^{2}$ and $S^{1}$. The neighborhood of the fiber over $P$ in the quotient (a compact interval), is the rational tangle $(\alpha, \alpha-1)$.([3].)

Let $C$ be a mirror cycle in $Q$. Let $N(D)$ be a neighborhood in $C$ of the set of corner points. Let $\Sigma$ be the surface obtained by the union of the fibres over the mirror points of $C-N(D)$, together with topological squares in the rational tangles at the corner points. Then $\varepsilon$ is 1 if $\Sigma$ is a Mœbius band, and 0 if it is an annulus.([3].)

The Euler class $e$ is the obstruction to extend a certain 2-fold partial section. It coincides with the number $-b$ in the signature of Seifert for Seifert manifolds.

The rational Euler class $e_{0}$ is defined by:

$$
e_{0}=e-\Sigma\left\{m(P) \frac{\beta(P)}{\alpha(P)} ; P \quad \text { a cone or corner point }\right\}
$$

where $m(P)=\frac{1}{2}$ if $P$ is a corner point, and $m(P)=1$ if $P$ is a cone point. The following result is well known:

Proposition 3.2.1. Let $Q$ be a 2-orbifold. Then $\chi(Q)=e_{0}(S T(Q))$.

Theorem 3.2.2. a) Figure 3.5 shows a surgery presentation of $S T(Q)$, where $Q$ is the hyperbolic orbifold consisting of the orientable surface of genus $g \geq 0, s$ cone points with isotropy group $C_{\alpha_{i}},(i=1, \ldots, s), m$ mirror edges $\ell_{1}, \ell_{2}, \ldots, \ell_{m}$, and $r_{j}$ corner points with isotropy group $\Delta_{2 \beta_{j n}},\left(n=1, \ldots, r_{j}\right)$ in each $\ell_{j},(j=1, \ldots, m)$. The singular orbits of the geodesic flow on $Q$ form the link $L_{1} \cup L_{2} \cup \ldots \cup L_{m}$, and every $L_{j}$ is a thorn.

b) Figure 3.6 shows a surgery presentation of $S T(Q)$, where $Q$ is the hyperbolic orbifold consisting of the non-orientable surface of genus $k \geq$ $0, s$ cone points with isotropy group $C_{\alpha_{i}},(i=1, \ldots, s), m$ mirror edges $\ell_{1}, \ell_{2}, \ldots, \ell_{m}$, and $r_{j}$ corner points with isotropy group $\Delta_{2 \beta_{j n}},\left(n=1, \ldots, r_{j}\right)$ in each $\ell_{j},(j=1, \ldots, m)$. The singular orbits of the geodesic flow on $Q$ form 
the link $L_{1} \cup L_{2} \cup \ldots \cup L_{m}$, and every $L_{j}$ is a thorn.

Proof. For $m=0, S T(Q)$ is the Seifert manifold

$$
M=\left(0, \underset{0, g}{n, k}:-e ;\left(\alpha_{1}, \alpha_{1}-1\right), \ldots,\left(\alpha_{s}, \alpha_{s}-1\right)\right)
$$

and Fig. 3.5 or 3.6 is a surgery presentation for it $([\mathbf{1 9}$, p. 146]).

Then it is enough to prove that Fig. 3.7 is a surgery presentation of $S T(U)$, where $U$ is a neighborhood in $Q$ of a mirror edge having $r$ corner points with isotropy groups $\Delta_{2 \beta_{1}}, \ldots, \Delta_{2 \beta_{r}}$, and to check that $L$ is the set of fixed points for the reflection.

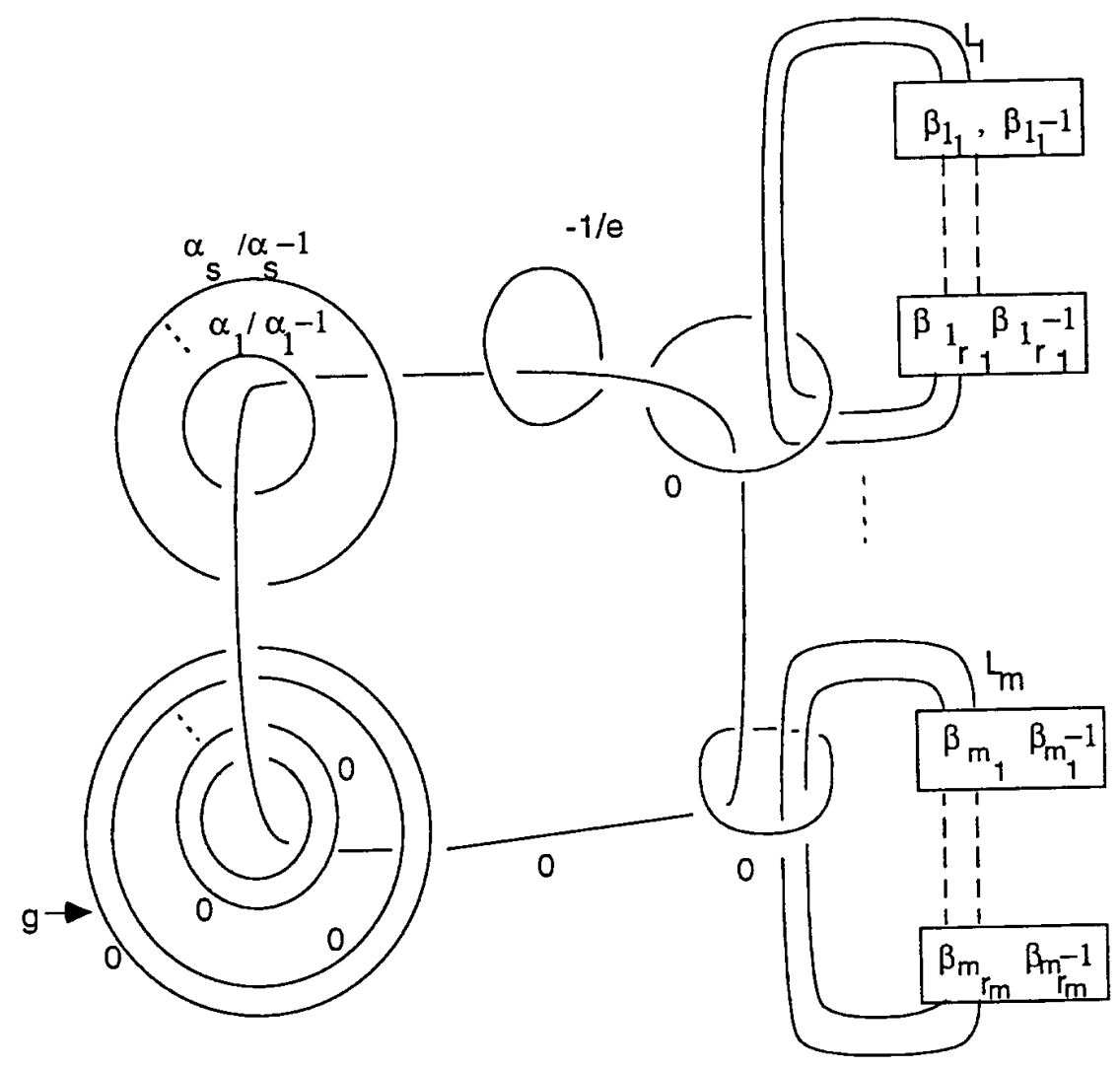

Figure 3.5. 


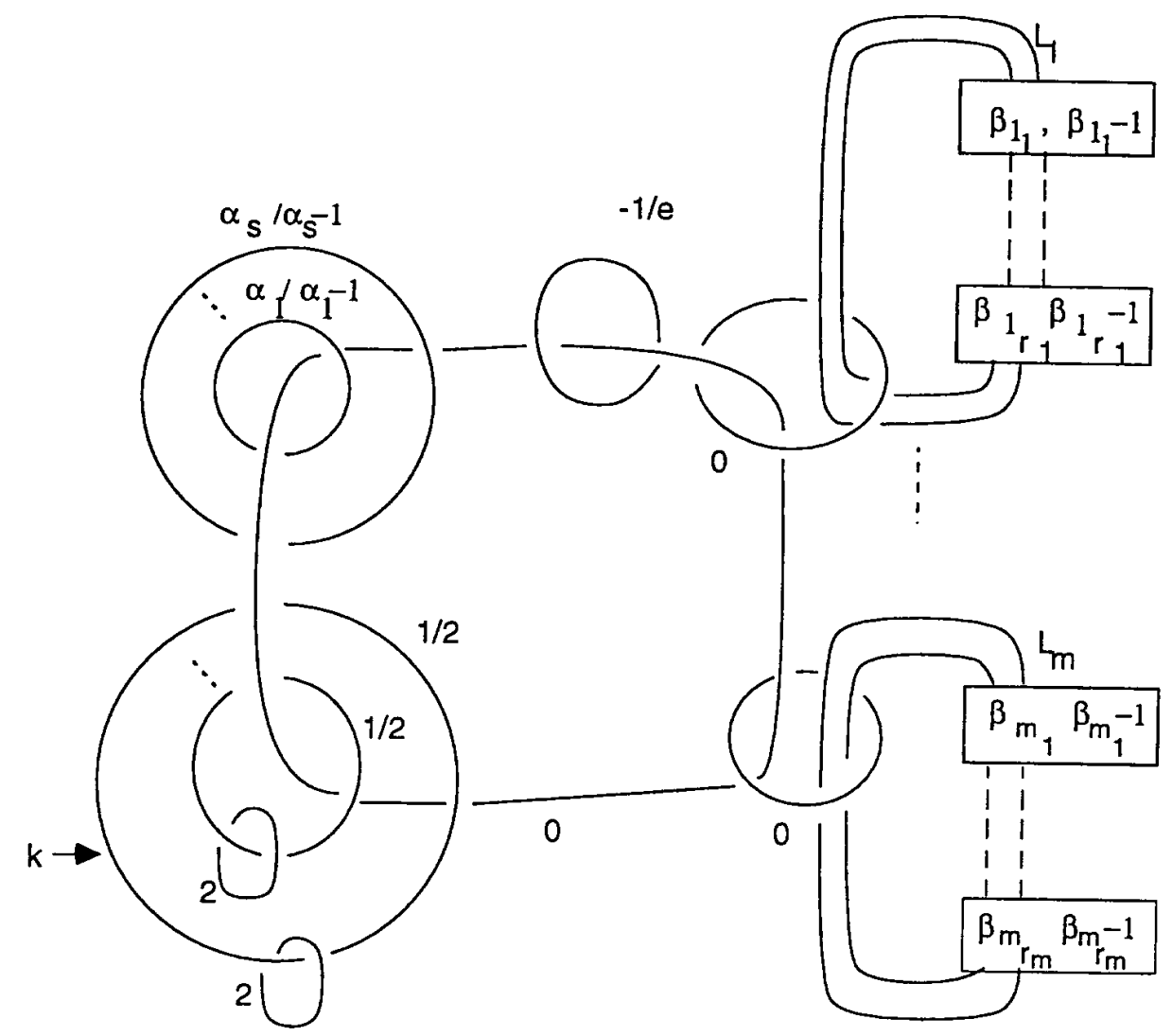

Figure 3.6.

Where $([\mathbf{1 7}\rceil,\lceil\mathbf{1 8}\rceil)$

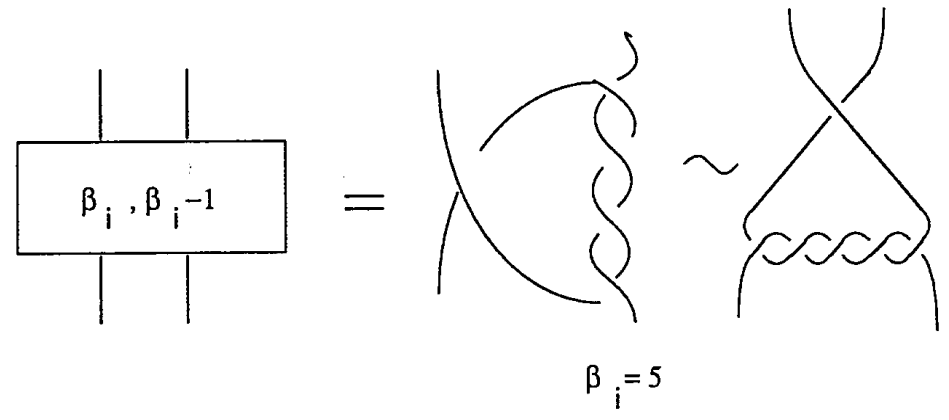

Let $m$ be a boundary component of $Q$. Then, each fiber $\{x\} \times S^{1}$ of the fibred torus $m \times S^{1}$ (Fig. 3.8a)) must be folded to a closed interval, and thus the torus $m \times S^{1}$ becomes a $I$-fibred anulus $A$. To do that, we first change the surgery instructions as it is shown in Fig. 3.8b). Fig. 3.8c) stands for the final result when there are no corner points in $m$. The boundary of $A$ forms the fixed points for the reflection. If there are corner points in $m$, and $P_{i}$ is 
one of them with isotropy group $\Delta_{2 \beta i}$, we must substitute a neighborhood of $P_{i} \times I$ by the rational tangle $\left(\beta_{i}, \beta_{i}-1\right)$. Therefore the final result is as in Fig. 3.7.

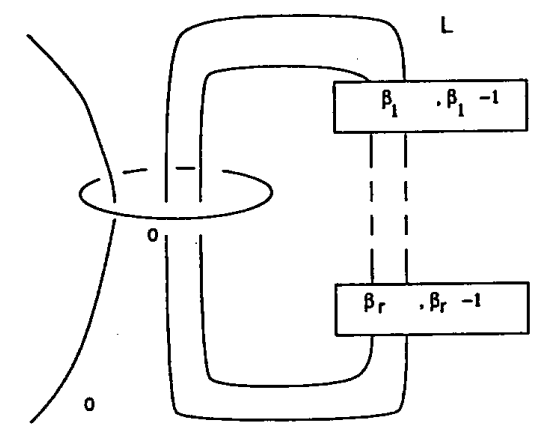

Figure 3.7.

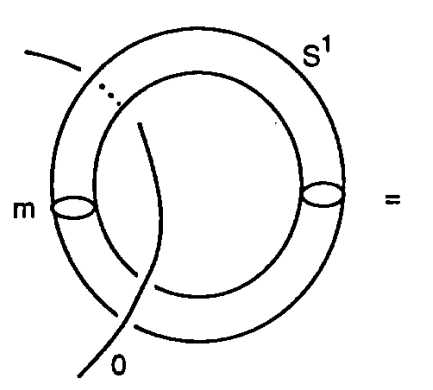

a)

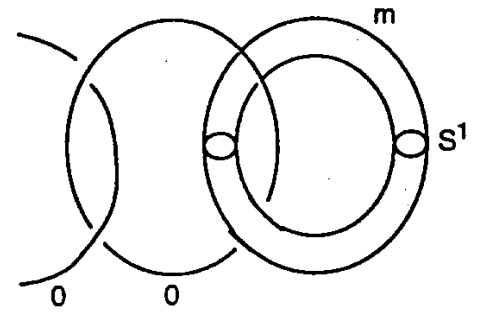

b)

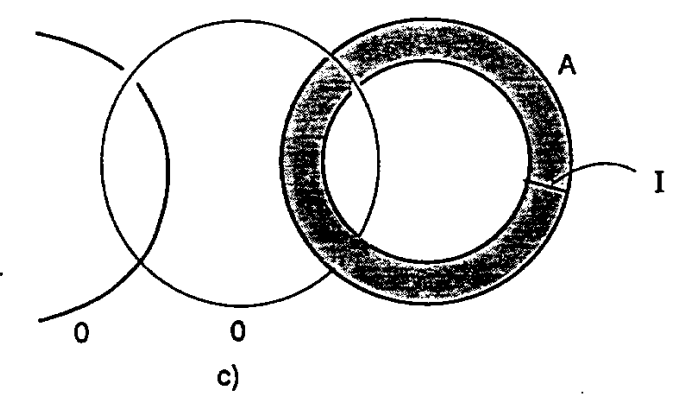

Figure 3.8.

$\S 4$. $V$-Anosov flows in $S^{3}$.

4.1. Geodesic flows of orbifolds, in $S^{3}$. The following theorem gives a family of $V$-Anosov flows in $S^{3}$. The flow is Anosov in the exterior of a Montesinos link $L$. The double cover of $S^{3}$ branched over $L$ is a 3 -manifold $M_{L}$ with an Anosov flow $\varphi_{M}$. This gives a structure of 3-orbifold to $S^{3}$ with $L$ as singular locus and where the isotropy group of each singular point is 
cyclic of order 2 . This structure on $S^{3}$ is the quotient of $M_{L}$ by an involution commuting with the flow.

Theorem 4.1.1. Let $L$ be the Montesinos link of Fig. 4.1, where $r, \alpha_{i}(i=$ $1, \ldots, r)$ are natural numbers such that $2+\sum_{i=1}^{r} \frac{1}{\alpha_{i}}<r$. Then there exists a $V$-Anosov flow in $S^{3}$ whose singular set is the link $L$.

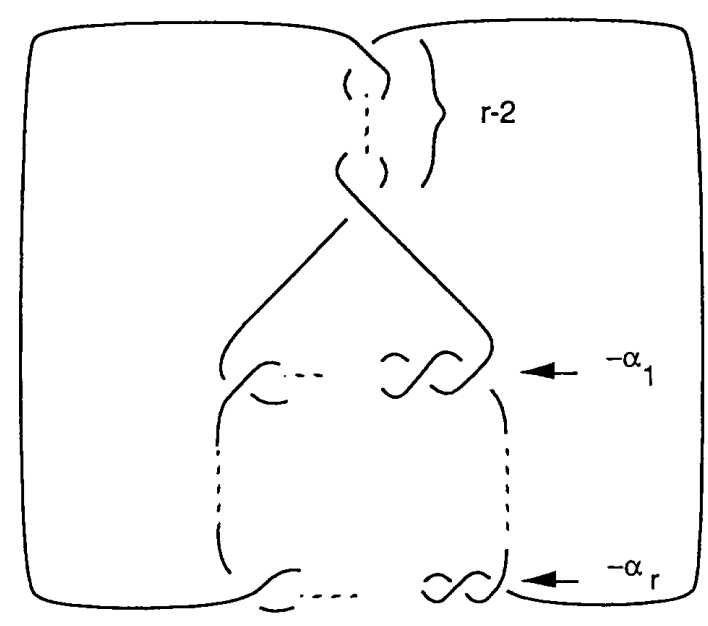

Figure 4.1.

Proof. Let $Q$ be the hyperbolic orbifold consisting of a disk $D$ with mirror boundary and $r$ corner points with isotropy groups $\Delta_{2 \alpha_{1}}, \ldots, \Delta_{2 \alpha_{r}}$, where $\alpha_{i} \in \mathbb{Z}$. We denote $Q$ by $D_{\alpha_{1} \alpha_{2} \ldots \alpha_{r}}$. Then $2+\sum_{i=1}^{r} \frac{1}{\alpha_{i}}<r$, because, the Euler characteristic of the (hyperbolic) $Q$ is negative.

To construct $S T(Q)$, we start considering the solid torus $D^{2} \times S^{1}$. In the boundary torus $\partial D^{2} \times S^{1}$ (set of fibers over the mirror boundary $\partial D$ ), each fiber $\{x\} \times S^{1}$ must be folded to an interval. That operation transforms $\partial D^{2} \times S^{1}$ in a $I$-fibred annulus $A$. The resulting underlying manifold is $S^{3}$. (Fig. 4.2.)

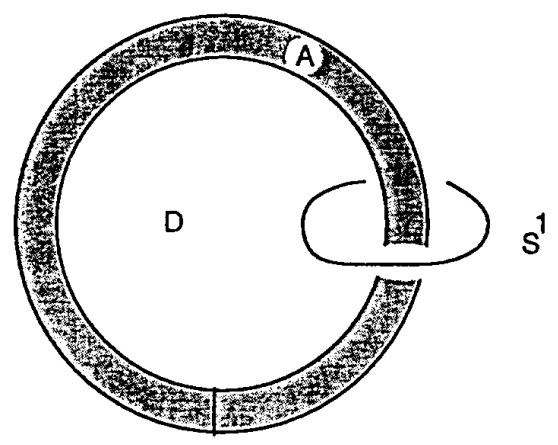

Figure 4.2. 
We have to replace a neighbourhood of $\left\{P_{i}\right\} \times I$ by a fibred tangle $\left(\alpha_{i}, \alpha_{i}-\right.$ 1 ), where $P_{i}$ is a corner point with isotropy group $\Delta_{2 \alpha_{i}}$. The underlying manifold is again $S^{3}$.

We compute the Euler class $e$ by the formula: $\chi(Q)=e_{0}$, where

$\chi(Q)=\frac{1}{2}\left(\sum_{i} \frac{1}{\alpha_{i}}-r+2\right) \quad$ and $\quad e_{0}=e-\frac{1}{2} \sum_{i} \frac{\alpha_{i}-1}{\alpha_{i}}=e-\frac{r}{2}+\frac{1}{2} \sum_{i} \frac{1}{\alpha_{i}}$.

This implies that $e=1$. One obtains $e=1$ by doing $(-1)$-surgery in a general fibre. Then Fig. 4.3 is a presentation of $S T(Q)$. Using Hempel trick [11] (Fig. 4.4) we obtain Fig. 4.5. As isotropy transforms the link of Fig. 4.5 in the link $L$. This shows that the underlying space of $S T(Q)$ is $S^{3}$. The geodesic flow of $S T(Q)$ is a $V$-Anosov flow and $L$ is the singular set of the flow because it is the set of fixed points for the action on $S T(\mathbb{H})$ of the subgroup $\Gamma$ of isometries of $\mathbb{H}^{2}$ defining $Q$.

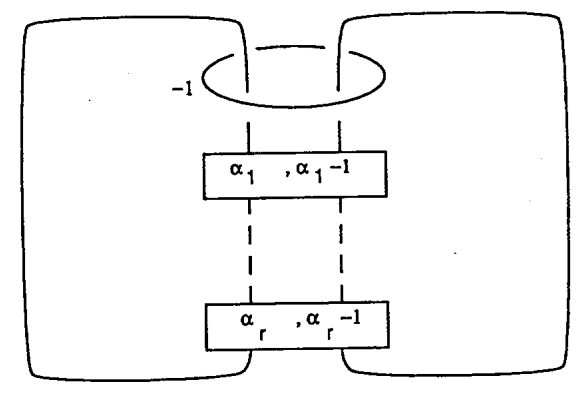

Figure 4.3.

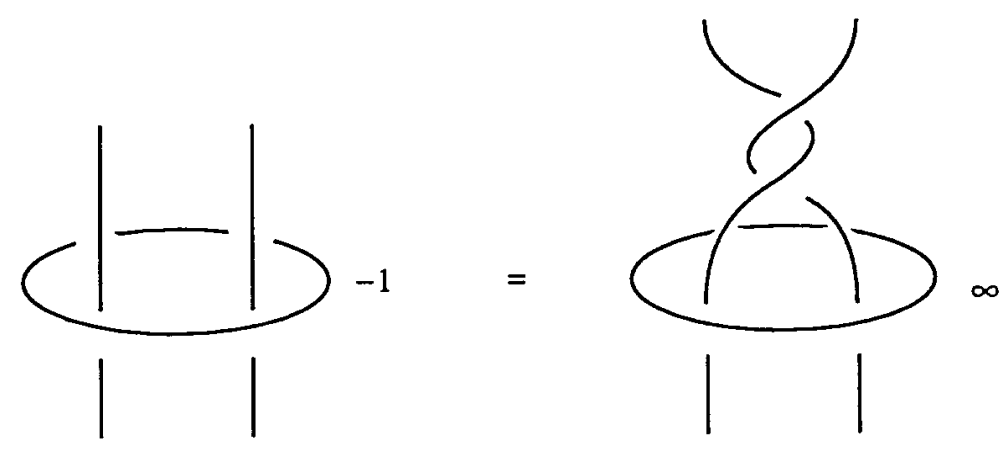

Figure 4.4.

Note that the thesis of the theorem could have also been obtained as a consequence of Theorem 3.2.2 and Kirby calculus. 
The manifold $M_{L}$, double cover of $S^{3}$ branched over $L$, has an Anosov flow invariant under the involution $u$ of $M_{L}$ such that $S^{3}=M_{L} / u$. Moreover $M_{L}$ is the Seifert manifold $\left(O, o, 0 \mid-2 ;\left(\alpha_{1}, \alpha_{1}-1\right), \ldots,\left(\alpha_{r}, \alpha_{r}-1\right)\right)$. This structures $S^{3}$ as a Seifert orbifold with singular locus of order 2 in $L$.

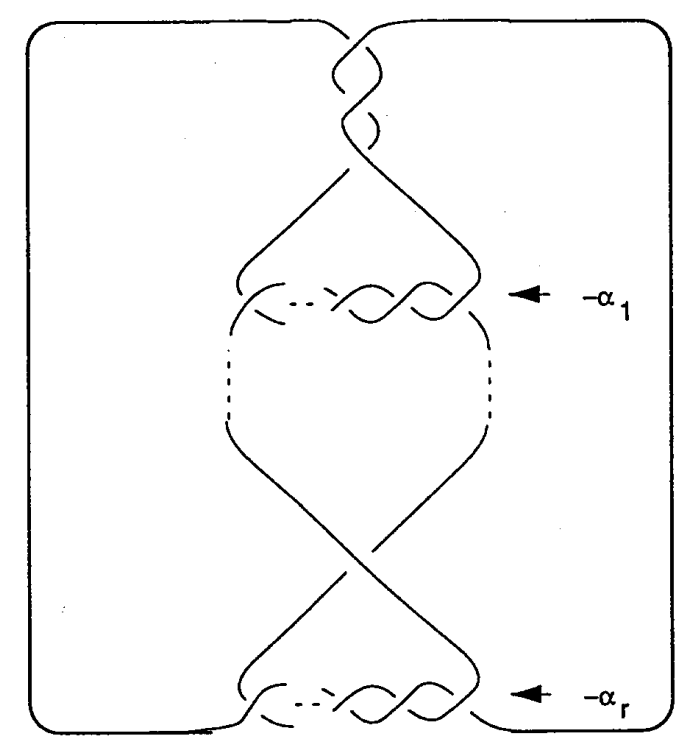

Figure 4.5.

4.2. Braided $V$-Anosov flows in $S^{3}$. We will construct a family of $V$ Anosov flows in $S^{3}$. Each of these will be the quotient of an Anosov suspension flow in certain torus bundle over $S^{1}$. The flow singularity is a certain 3 -braid link with or without its axis.

Every element $B$ of $S L(2, \mathbb{Z})$ is the matrix of a linear map $f_{B}$ in $\mathbb{R}^{2}$ leaving $\mathbb{Z}^{2}$ invariant. It induces a diffeomorphism, still called $f_{B}$, in the quotient manifold $F_{1}=\mathbb{R}^{2} / \mathbb{Z}^{2}$. The diffeomorphism $f_{B}$ is the diffeomorphism on $F_{1}$ associated to $B$. Note that the point $x_{0} \in F_{1}$, which is the orbit of $(0,0)$, is a fixed point of the diffeomorphism $f_{B}$ of $F_{1}$.

Let $f$ be an Anosov diffeomorphism on the torus $F_{1}$. It is well known that there exists an Anosov matrix $A_{f} \in S L(2, \mathbb{Z})$ such that $f$ is isotopic to the diffeomorphism associated to $A_{f}$.

Every Anosov matrix is similar over the integers to a matrix with all its entries positive [26].

Every matrix $B=\left(\begin{array}{ll}a & b \\ c & d\end{array}\right) \in S L(2, \mathbb{Z})$, where $a, b, c, d>0$, is hyperbolic (because $a+d>2$ ), and it is the image by the reduced Burau representation 
$\rho$, of a hyperbolic pure 3-braid

$$
\gamma_{0}=\bar{\sigma}_{1}^{p_{1}} \sigma_{2}^{q_{1}} \ldots \bar{\sigma}_{1}^{p_{r}} \sigma_{2}^{q_{r}} \quad r \geq 1, \quad p_{i}, q_{i} \in \mathbb{N} .
$$

Recall that $\rho\left(\bar{\sigma}_{1}\right)=\left(\begin{array}{ll}1 & 0 \\ 1 & 1\end{array}\right)$ and $\rho\left(\sigma_{2}\right)=\left(\begin{array}{ll}1 & 1 \\ 0 & 1\end{array}\right)$. ([2].)

Geometrically, $B$ represents also the following two diffeomorphisms:

(i) A diffeomorphism on a one-puntured sphere $F_{0,1}$ with three distinguished points as follows. Consider $F_{0,1}$ as the orbit space $\left(\mathbb{R}^{2}-\mathbb{Z}^{2}\right) / G$, where $G$ is the group generated by $\pi$-rotation of $\mathbb{R}^{2}$ about the points with coordinates $\left(\frac{m}{2}, \frac{n}{2}\right), m, n \in \mathbb{Z}$. The action $g_{B}$ of $B \in S L(2, \mathbb{Z})$ on $F_{0,1}$ is the quotient of the action of $B$ on $\mathbb{R}^{2}$. The three distinguished points $x_{1}, x_{2}, x_{3}$ in $F_{0,1}$ are the orbits of $\left(\frac{1}{2}, 0\right),\left(0, \frac{1}{2}\right)$ and $\left(\frac{1}{2}, \frac{1}{2}\right)$. Note that the set $\left\{x_{1}, x_{2}, x_{3}\right\}$ is invariant by $g_{B}$.

(ii) A diffeomorphism on a one-puntured torus $F_{1,1}$. This is the restriction $h_{B}$ of $f_{B}$ to $F_{1,1}$ assuming that the punctured point of $F_{1,1}$ is $x_{0}$.

Let $N_{B}^{o}$ be the mapping torus over $F_{0,1}$ with monodromy $g_{B} \cdot N_{B}^{0}=F_{0,1} \times$ $[0,1] /(x, 1) \equiv\left(g_{B}(x), 0\right)$. Topologically $N_{B}^{o}$ is an open solid torus, containing every closed braid $\widehat{\gamma}$, where $\gamma$ is a geometric 3-braid represented by $B$ (see [2, p. 40]). Fig. 4.6 shows the geometric 3 -braid generators.
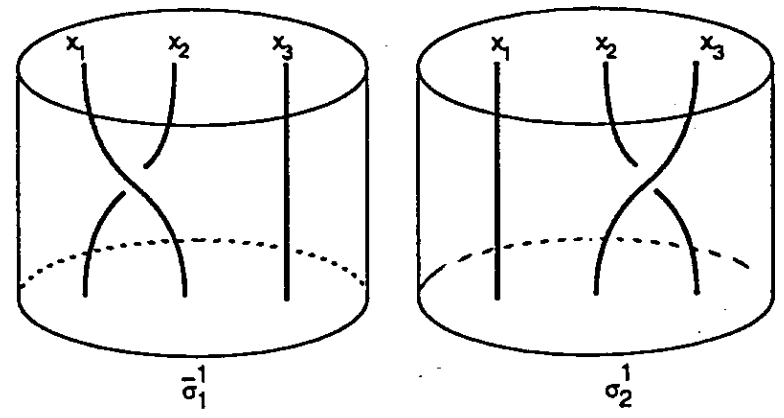

Figure 4.6.

Let $M_{B}^{o}$ be the mapping torus over the once-punctured torus $F_{1,1}$ with monodromy $h_{B} . M_{B}^{o}=F_{1,1} \times[0,1] /(x, 1) \equiv\left(h_{B}(x), 0\right)$.

Then there exists a map $p: M_{B}^{o} \longrightarrow N_{B}^{0}$ which is a double covering branched along the closed braid represented by $B$.

To prove it note that both manifolds, $M_{B}^{o}$ and $N_{B}^{o}$ have an open book structure with deleted binding, and monodromies $h_{B}, g_{B}$ associated to $B$. The map $p$ on each sheet of $M_{B}^{o}$ (once-punctured torus $F_{1,1}$ ) is the double cover of the sheet of $N_{B}^{o}$ (once-punctured sphere $F_{0,1}$ ) branched over $\left\{x_{0}, x_{1}, x_{2}\right\}$. Then the branching set of $p$ forms a closed braid $\widehat{\gamma}$, whose preimage is a closed orbit of the suspension flow $\varphi_{M}$ in $M_{B}^{o}$. The flow $\varphi_{M}$, being 
the suspension flow by an Anosov diffeomorphism, is an Anosov flow. The suspension flow $\varphi_{N}$ in $N_{B}^{o}$ is a $V$-Anosov flow, it is the projection by $p$ of the Anosov flow $\varphi_{M}$. The singular set of $\varphi_{N}$ is $\widehat{\gamma}$. The compactification of $N_{B}^{o}$ with the axis $E_{\gamma}$ of the braid $\widehat{\gamma}$ is $S^{3}$. We want the extension of the flow $\varphi_{M}$ to $S^{3}$ to be a $V$-Anosov flow. That depends on the braid $\gamma$ as follows.

For the hyperbolic pure 3-braid

$$
\gamma_{0}=\bar{\sigma}_{1}^{p_{1}} \sigma_{2}^{q_{1}} \ldots \bar{\sigma}_{1}^{p_{r}} \sigma_{2}^{q_{r}} \quad r \geq 1, \quad p_{i}, q_{i} \in \mathbb{N},
$$

such that $\rho\left(\gamma_{0}\right)=B$ we can isotope $h_{B}$ slightly, in a neighbourhood of $(m, n), m, n \in \mathbb{Z}$, so that $h_{B}$ is the identity near $(0,0)$ and such that every meridian curve of the axis $E_{\gamma_{0}}$ is an orbit of the suspension flow $\varphi_{M}$. Then the extension of the flow $\varphi_{M}$ to $S^{3}$ has the axis $E_{\gamma_{0}}$ as a set of fixed points, so that it is not a $V$-Anosov flow.

Note that in the Burau representation $\rho$ :

$$
\begin{aligned}
\sigma_{1} \sigma_{2} \sigma_{1} & \longrightarrow\left(\begin{array}{cc}
1 & 0 \\
-1 & 1
\end{array}\right)\left(\begin{array}{ll}
1 & 1 \\
0 & 1
\end{array}\right)\left(\begin{array}{cc}
1 & 0 \\
-1 & 1
\end{array}\right)=\left(\begin{array}{cc}
0 & 1 \\
-1 & 0
\end{array}\right) \\
\left(\sigma_{1} \sigma_{2} \sigma_{1}\right)^{2} & \longrightarrow\left(\begin{array}{cc}
-1 & 0 \\
0 & -1
\end{array}\right) .
\end{aligned}
$$

Then $\Delta^{4}=\left(\sigma_{1} \sigma_{2} \sigma_{1}\right)^{4}$ is represented by the matrix $\left(\begin{array}{ll}1 & 0 \\ 0 & 1\end{array}\right)$, whose effect in a neighbourhood of $(0,0)$ is a $2 \pi$-rotation. Thus $\rho\left(\Delta^{ \pm 4} \gamma_{0}\right)=B$. Let $\gamma=\Delta^{ \pm 4} \gamma_{0}$. In the $S^{3}$ which is the compactification of $N_{B}^{o}$ with the axis $E_{\gamma}$ of the braid $\hat{\gamma}$, the extension of the flow $\varphi_{M}$ is a $V$-Anosov flow, where the axis is an ordinary orbit and the singular set of the flow is $\hat{\gamma}$.

The above flow can be obtained directly from $\gamma_{0}$ as follows. Let $L$ and $M$ be the canonical longitude and meridian, respectively, of the axis of the closed braid $\widehat{\gamma}_{0}$. If we compactify $N_{B}^{o}$ in such a way that the meridian of the added solid torus $Q$ is $Q= \pm 2 L+M$, the result is again $S^{3}$. The extended flow has the closed braid corresponding to $\Delta^{ \pm 4} \gamma_{0}$ as a thorn, and now the axis is a regular periodic orbit.

Other possibility is to consider $Q= \pm L+M$. In this case the manifold is still $S^{3}$, and the extended flow is a $V$-Anosov flow with singular set the closed braid corresponding to $\Delta^{ \pm 2} \gamma_{0}$ together with its axis.

We have proved the following theorem

Theorem 4.2.1. Let $\gamma$ be a hyperbolic braid:

$$
\gamma=\bar{\sigma}_{1}^{p_{1}} \sigma_{2}^{q_{1}} \ldots \bar{\sigma}_{1}^{p_{r}} \sigma_{2}^{q_{r}} \quad r \geq 1, \quad p_{i}, q_{i} \in \mathbb{N} .
$$

There exists a singular flow in $S^{3}$, with singular set $L$, where $L$ is 
a) the closed braid corresponding to $\Delta^{ \pm 4} \gamma$, or

b) the closed braid corresponding to $\Delta^{ \pm 2} \gamma$ and its axis.

Corollary 4.2.2. No rational knot $K$ is isotopic to the closed 3-braid $L$ corresponding to $\Delta^{ \pm 4} \bar{\sigma}_{1}^{p_{1}} \sigma_{2}^{q_{1}} \ldots \bar{\sigma}_{1}^{p_{r}} \sigma_{2}^{q_{r}}$, where $r \geq 1, p_{i}, q_{i} \in \mathbb{N}$.

Proof. The knot $L$ is the thorn of a $V$-Anosov flow. Then the double cover of $S^{3}$ branched over $L$ has an Anosov flow. Margulis [16] proved that in a manifold having an Anosov flow, the fundamental group has exponential growth. (A finitely generated group $G$ is said to have exponential growth if given a finite set of generators, there exist two positive real numbers $a$ and $A$ such that $\Gamma(n)>A e^{a n}$, where $\Gamma(n)$ is the number of different elements of $G$ of word-length $\leq n$.) But the double cover of $S^{3}$ branched over a rational knot is a lens space, which has finite fundamental group, hence does not have exponential growth.

4.3. Relation between the set of thorns of geodesic flows and the set of thorns of braided $V$-Anosov flows in $S^{3}$. The following examples show that the set of thorns of the geodesic flows obtained in 4.1 is different from the set of thorns of the braided $V$-Anosov flows obtained in 4.2.

1) Let $\gamma=\Delta^{-4} \bar{\sigma}_{1}^{3} \sigma_{2} \bar{\sigma}_{1} \sigma_{2}$. (Fig. 4.7 shows that $\widehat{\gamma}$ is the knot $10_{152}[\mathbf{2 3}]$.) Theorem 4.2.1 affirms that the knot $10_{152}$ is the thorn of a $V$-Anosov flow $\varphi$ in $S^{3}$. The double cover of $S^{3}$ branched over the knot $10_{152}$ is a graph manifold, which is not a Seifert manifold. Then the flow $\varphi$ in $S^{3}$ is not a geodesic flow.

2) Let $\gamma=\Delta^{-2} \bar{\sigma}_{1} \sigma_{2}$. Consider the link $L$ composed by the closed braid $\widehat{\gamma}$ and its axis. Theorem 4.2.1 shows that $L$ is the singular set of a $V$-Anosov flow $\varphi$ in $S^{3}$. Fig. 4.8 shows the isotropy transforming $L$ into the closed braid $\widehat{\gamma}^{\prime}$ and its axis, where $\gamma^{\prime}=\bar{\sigma}_{1}^{5} \bar{\sigma}_{2}$. This link is the Montesinos link of Theorem 4.1.1 for $r=3, \alpha_{1}=2, \alpha_{2}=4, \alpha_{3}=5$. (Fig. 4.9.) Therefore $L$ is also the singular set of the geodesic flow of the orbifold $D_{245}$. 

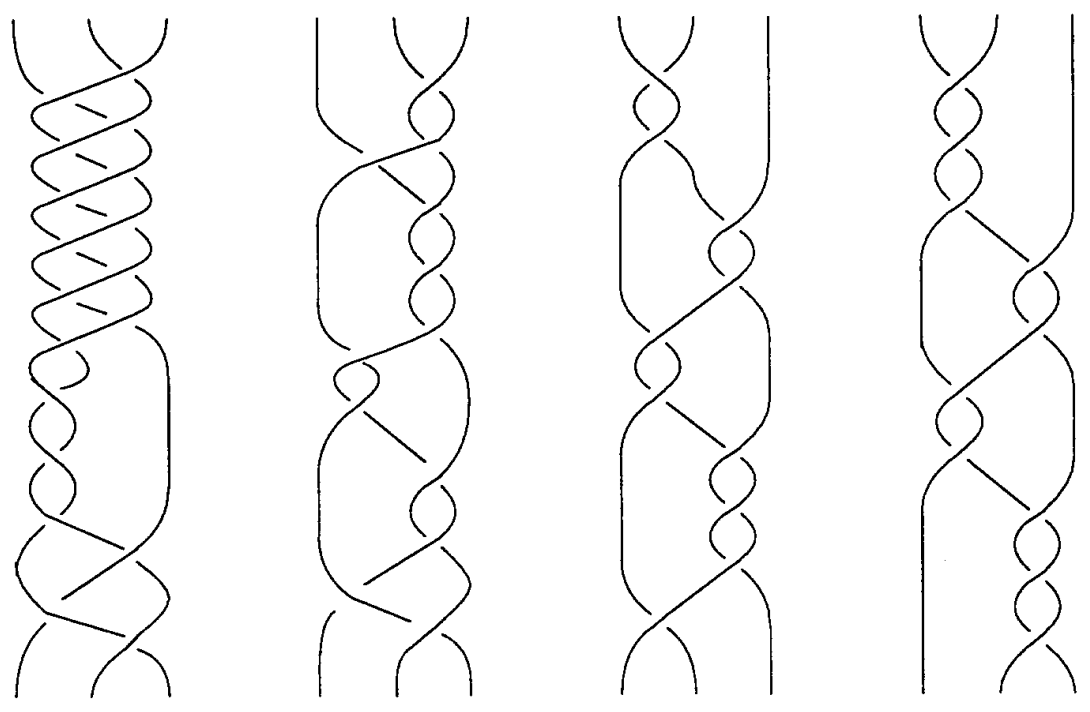

Figure 4.7.
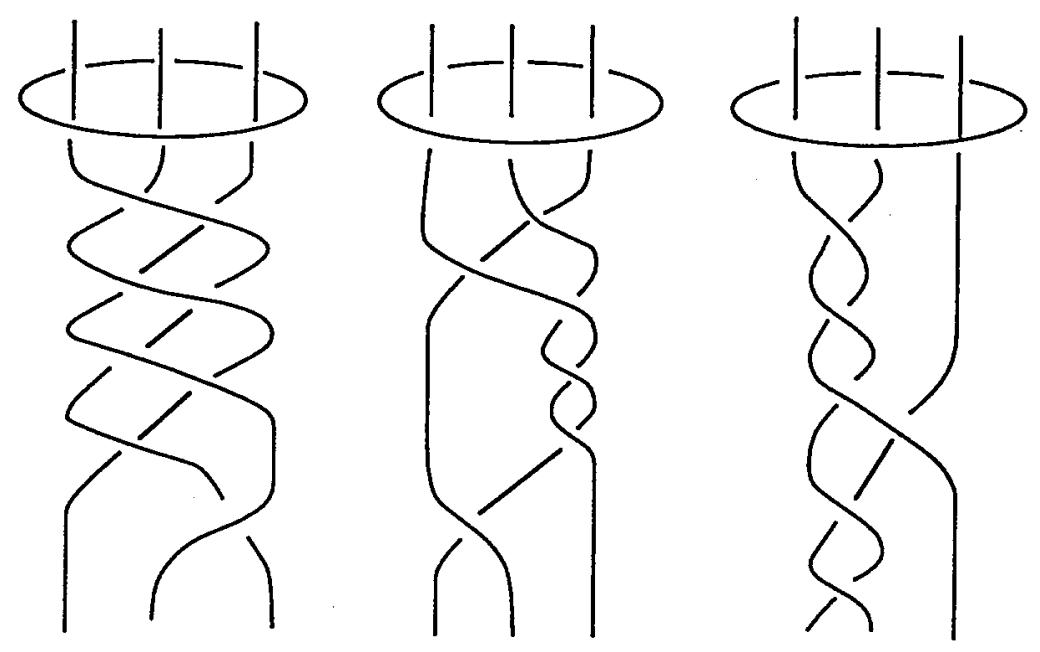

Figure 4.8.

3) The singular set of the geodesic flow of the orbifold $D_{222222}$ is a 6component link $L$. Then it can not be a 3 -braid or a 3 -braid together with its axis. Therefore $L$ is not the singular set of a braided $V$-Anosov flow. 


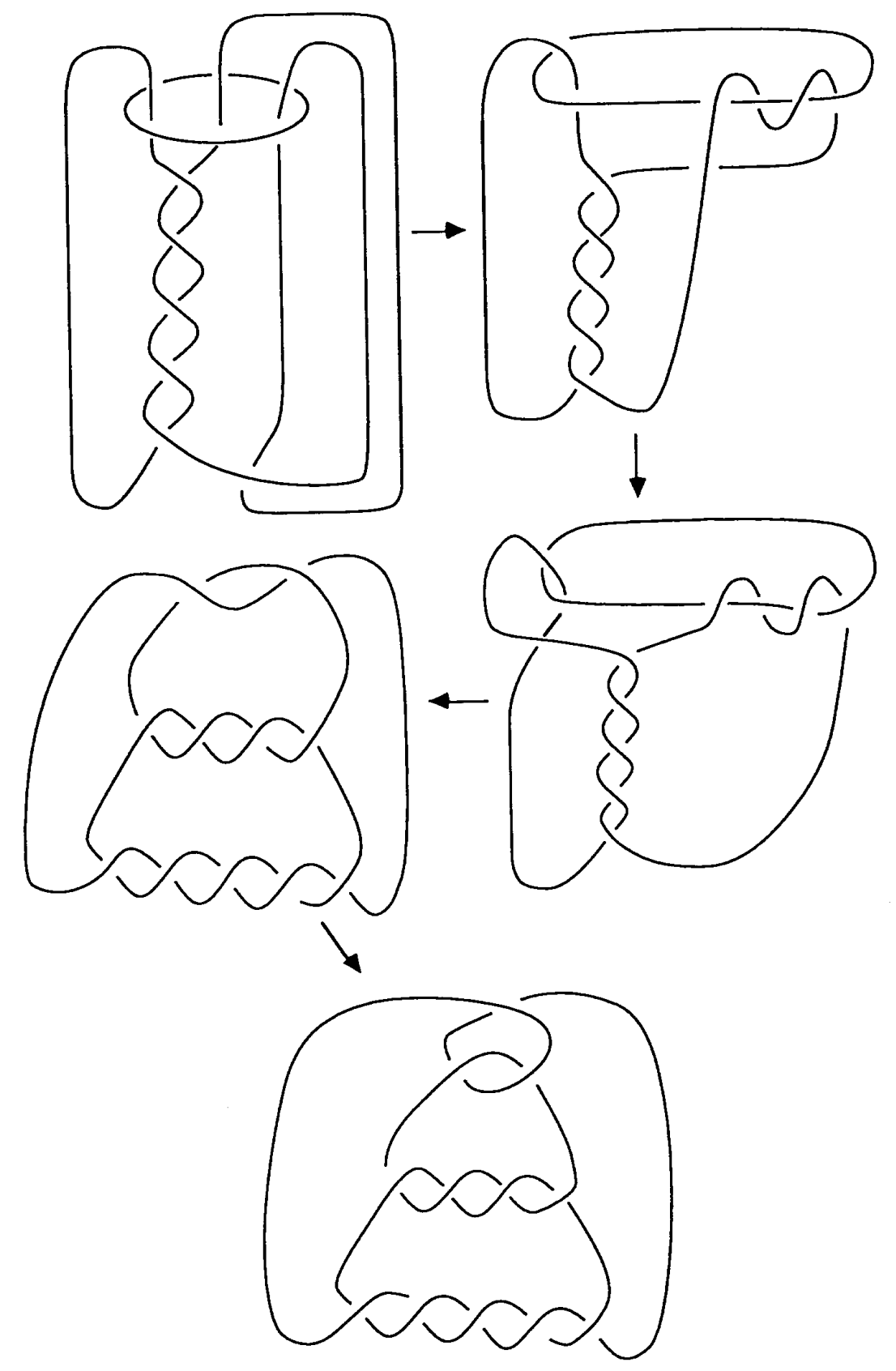

Figure 4.9. 
4.4. New $V$-Anosov flows from old ones. The following transformations produce new $V$-Anosov flows.

1) Surgery. A periodic orbit $\gamma$ of a $V$-Anosov flow in a 3 -manifold $M^{3}$ has a well-defined pseudo-framing associated to the flow, this is the intersection of the boundary of a tubular neighbourhood $N(\gamma)$ of $\gamma$, with the stable leaf $W^{S}(\gamma)$ containing $\gamma$.

If $\gamma$ is a thorn or a regular periodic orbit such that $N(\gamma)$ intersects $W^{S}(\gamma)$ in an annulus, this pseudo-framing defines coordinates in the boundary torus of $N(\gamma)$ as follows. $M=\partial D$, where $D$ is a meridian disk of $N(\gamma)$, and $L$ equals one of the two components of $\partial N(\gamma) \cap W^{S}(\gamma)$.

If $\gamma$ is a regular periodic orbit such that $N(\gamma)$ intersects $W^{S}(\gamma)$ in a Mœbius band $B$, we choose $L$ such that $\partial B=2 L+M$, where $M=\partial D$, and $D$ is a meridian disk of $N(\gamma)$.

In both cases we orient $M$ and $L$ such that $L \sim \gamma$ oriented by the flow, and $M . L=+1$ in the orientation of $M^{3}$.

Then "admissible Dehn surgery" in $M^{3}$ along $\gamma$, produces a new manifold $N^{3}$ with a $V$-Anosov flow. We say that a Dehn surgery along $\gamma$ is admissible if the new periodic orbit $\gamma^{\prime}$ is regular or thorn. (Recall Construction 2.1.1.)

In particular, if $M^{3}=S^{3}$ and $\gamma$ is the unknot, $N^{3}$ is still $S^{3}$, with a new $V$-Anosov flow.

2) Covering. Any covering of a 3-manifold with a $V$-Anosov flow branched over a set of thorns with branching indices 1 or 2 , induces a $V$-Anosov flow in the covering. Here we consider some particular cases.

i) Let $\varphi$ be a $V$-Anosov flow in $S^{3}$, and let $\gamma_{1}$ and $\gamma_{2}$ be thorns of $\varphi$ such that $\gamma_{1}$ and $\gamma_{2}$ are unlinked and unknotted. $S^{3}$ is the covering of $S^{3}\left(p: S^{3} \longrightarrow S^{3}\right)$ branched over $\gamma_{1} \cup \gamma_{2}$ defined by the monodromy:

$w\left(m_{1}\right)=(12)(34) \cdots(2 k-12 k)$ and $\quad w\left(m_{2}\right)=(23)(45) \cdots(2 k 2 k+1)$,

where $m_{1}$ and $m_{2}$ are the meridians of $\gamma_{1}$ and $\gamma_{2}$, respectively.

The branching indices are 1 and 2. Then there is a $V$-Anosov flow $\psi$ in the cover $S^{3}$ such that the singular set is composed by the pseudo-branching (preimage of $\gamma_{1}$ and $\gamma_{2}$ with branching index 1) and the preimage of the singular set of $\varphi$ different from $\gamma_{1}$ and $\gamma_{2}$.

In particular we can apply this procedure to the double cover of $S^{3}$ branched over an unknotted thorn.

ii) Next we construct an Anosov flow on a manifold $M$, such that there exist a periodic orbit such that the component of the intersection of it stable submanifold with a regular neighbourhood containig $p^{-1}(\gamma)$ is a Mœbius band. Consider the $V$-Anosov flow in $S^{3}$ whose singular set is the Montesinos link $L$ of Fig. 4.10. It is the geodesic flow of the orbifold $D_{444}$. 


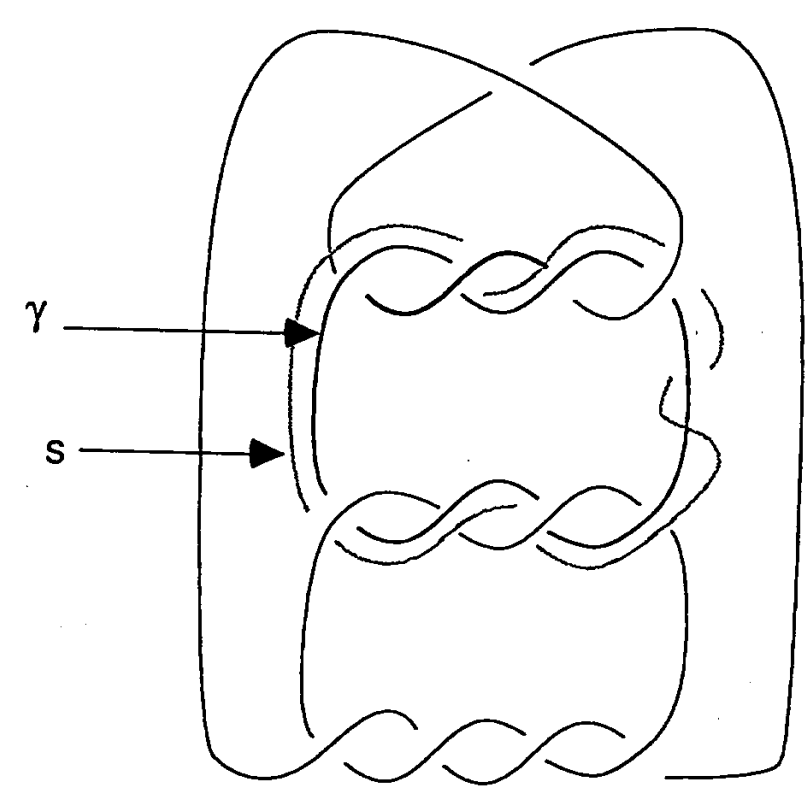

Figure 4.10.

The curve $s$ is the intersection of the stable submanifold of the thorn $\gamma$ with the boundary of a tubular neighbourhood of $\gamma$. Let $p: M \longrightarrow S^{3}$ be the double covering of $S^{3}$ branched over $L$. Then $p^{-1}(\gamma)$ is an ordinary orbit of the Anosov flow induced in $M$, such that the component of the intersection of it stable submanifold with a regular neighbourhood containig $p^{-1}(\gamma)$ is a Mœbius band. This is because $p^{-1}(\gamma)$ is connected. (Compare Construction 2.2.1 and Note 1 of Corollary 2.2.3.)

\section{$\S 5$. Singular Anosov Flows in 3-manifolds.}

5.1. The universal orbifolds $(L, 2)$. Let $(L, s)$ denote $S^{3}$ structured as a 3 -orbifold with singular locus a link $L$ and isotropy cyclic of order $s \in \mathbb{N}$.

Definition 5.1.1. The orbifold $(L, s)$ is a universal orbifold if every 3manifold is a cover of $S^{3}$ branched over $L$ such that the branching indices divide $s$.

In this section we will obtain some universal orbifolds $(L, 2)$. The interest on universal orbifolds with isotropy cyclic of order 2 was pointed out by Thurston [25]. We will use them in Section 5.2, to obtain singular Anosov flows in 3-manifolds.

Definition 5.1.2. Let $p: M^{3} \longrightarrow N^{3}$ be a finite covering branched over a link $L$. Let $L^{\prime}$ be a sublink of $L$. The least common multiple of the branching indices of the points of $p^{-1}\left(L^{\prime}\right)$ will be called branching index of $L^{\prime}$, and will 
be denoted by $b\left(L^{\prime}\right)$. The branching index of $p$ is $b(L)$.

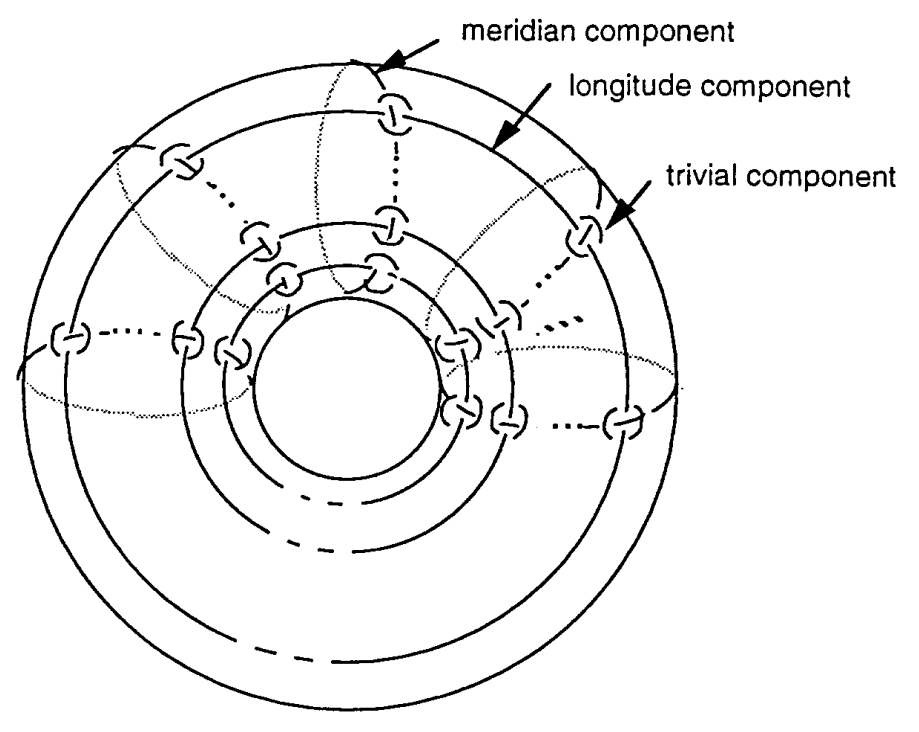

Figure 5.1.

Let $L_{m, n}$ be the link in $S^{3}$ showed in Fig. 5.1, where $m$ is the number of meridian components of $L_{m, n}$ and $n$ is the number of longitude components of $L_{m, n}$.

Theorem 5.1.3. Let $M^{3}$ be a closed oriented 3-manifold. Then there exist two natural numbers $m$ and $n$, and a covering map $p: M^{3} \longrightarrow S^{3}$ branched over the link $L_{m, n}$ such that the branching index of $p$ is 2 .

Proof. [14, proof of Theorem 1.1].

Note that $L_{m, n}$ is placed in such a way that it remains invariant under the action of $\mathbb{Z}_{m} \times \mathbb{Z}_{n}$ in $S^{3}$ generated by a $2 \pi / m$-rotation $r_{m}$ around the core $c_{m}$ of the unbounded solid torus, and by a $2 \pi / n$-rotation $r_{n}$ around the core $c_{n}$ of the bounded solid torus (this is the main idea of Thurston in [25]).

The $2 \pi / m$-rotation $r_{m}$ generates the group of covering transformations of an $m$-fold cyclic covering $f_{m}: S^{3} \longrightarrow S^{3}$, whose branching set is $f_{m}\left(c_{m}\right)$. We can modify $f_{m}$ into $f_{m}^{\prime}: S^{3} \longrightarrow S^{3}$, branched along two curves $c_{1}, c_{2}$ parallel to $f_{m}\left(c_{m}\right)$ and not linking each other. The modification takes place in a regular neighbourhood of $f_{m}\left(c_{m}\right)$, outside of which the covering remains cyclic. Fig. 5.2 shows the branching set and the monodromy of both coverings (see [20]). Note that $f_{m}^{\prime}$ is a covering with branching index 2. Let $f_{n}^{\prime}$ be the analogous branched covering constructed from $f_{n}$. 


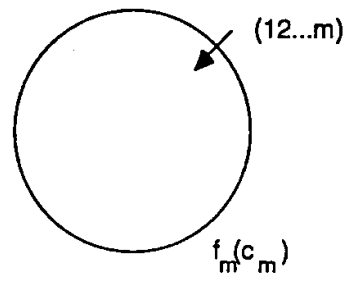

a) $f_{m}$

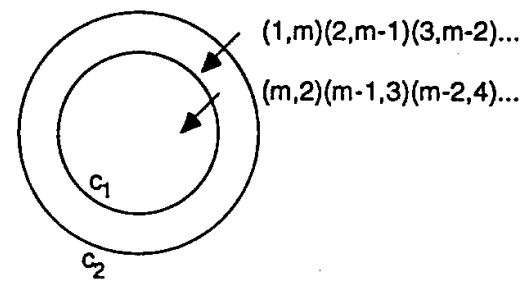

b) $\mathrm{f}_{\mathrm{m}}$

Figure 5.2.

The composition $p_{1}=f_{m}^{\prime} \circ p$ is a covering map from $M^{3}$ to $S^{3}$ branched over the link $f_{m}^{\prime}\left(L_{m, n}\right)$ with branching index 2 . The $2 \pi / n$-rotation $r_{n}$ around the core of the solid torus permutes the components of the link $f_{m}^{\prime}\left(L_{m, n}\right)$. Then the composition $p_{2}=f_{n}^{\prime} \circ p_{1}$ is a covering map from $M^{3}$ to $S^{3}$ branched over the link $L_{1}=f_{n}^{\prime}\left(f_{m}^{\prime}\left(L_{m, n}\right)\right)$ with branching index 2. (See Fig. 5.3.)

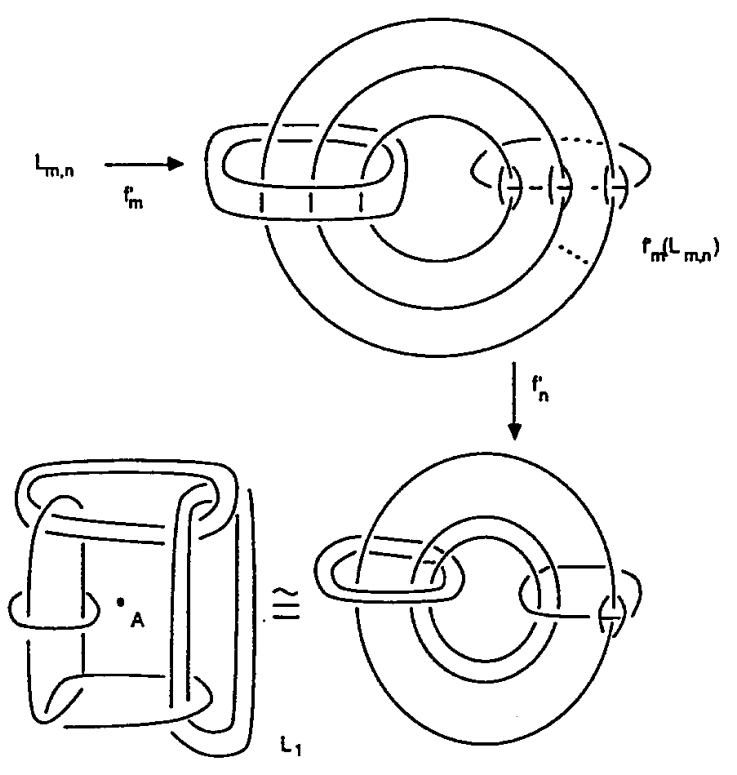

Figure 5.3.

Adding new components to $L_{1}$ we obtain a link invariant for the $\pi$-rotation around the axis passing through the point $A$ (Fig. 5.4). This produces a covering map $p_{3}: M^{3} \longrightarrow S^{3}$, branched over the link $L_{2}$, with branching index 2 . 


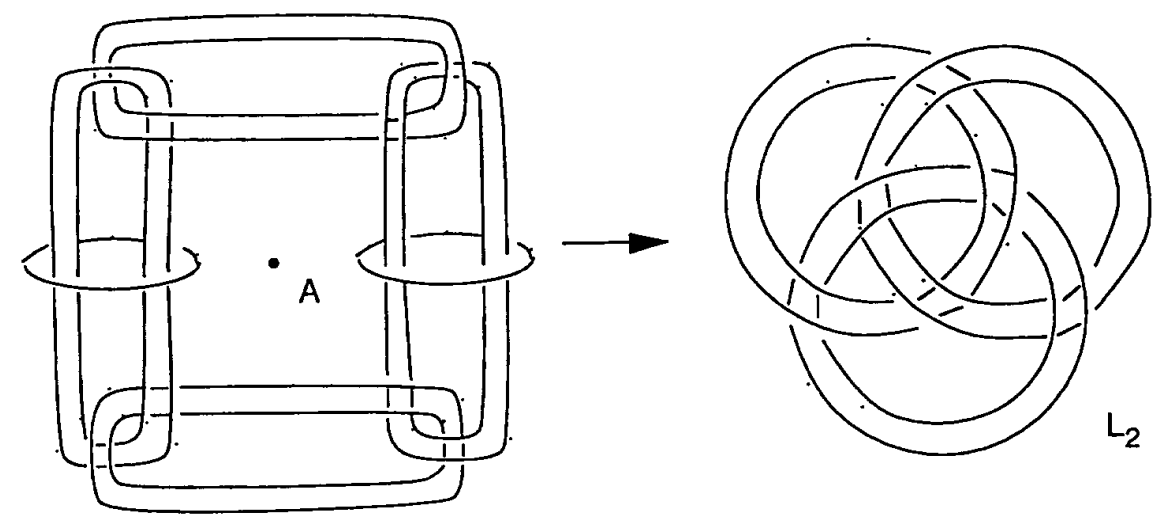

Figure 5.4.

Remark. Using the approach to obtain universal links given in [13] and the same ideas as above we obtain $L_{3}$ and $L_{4}$. (Fig. 5.5.)
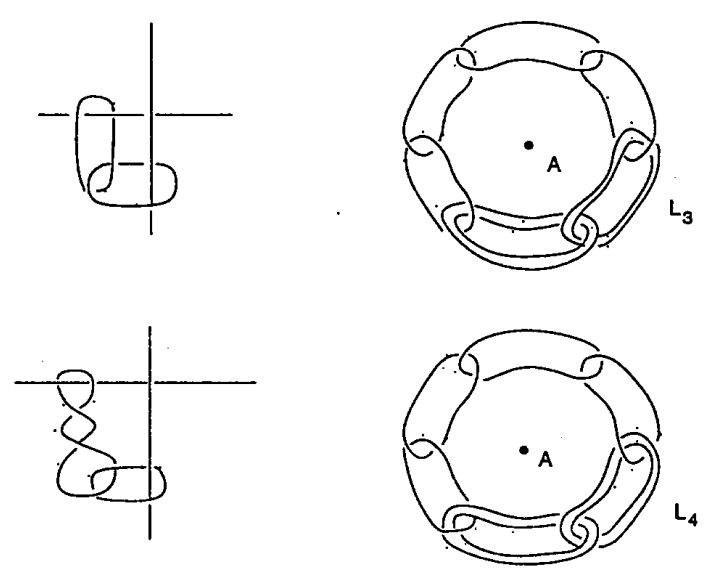

Figure 5.5.

Adding components to the links $L_{3}$ and $L_{4}$ so that they become symmetric with respect to the $2 \pi / 3$-rotation (or $2 \pi / 6$-rotation) around the axis passing through $A$ and using $f_{3}^{\prime}$ for $L_{3}$ and $f_{2}^{\prime}, f_{3}^{\prime}, f_{6}^{\prime}$ for $L_{4}$ we obtain again $L_{2}$, and $L_{5}, L_{6}, L_{7}$. (Fig. 5.6.) 

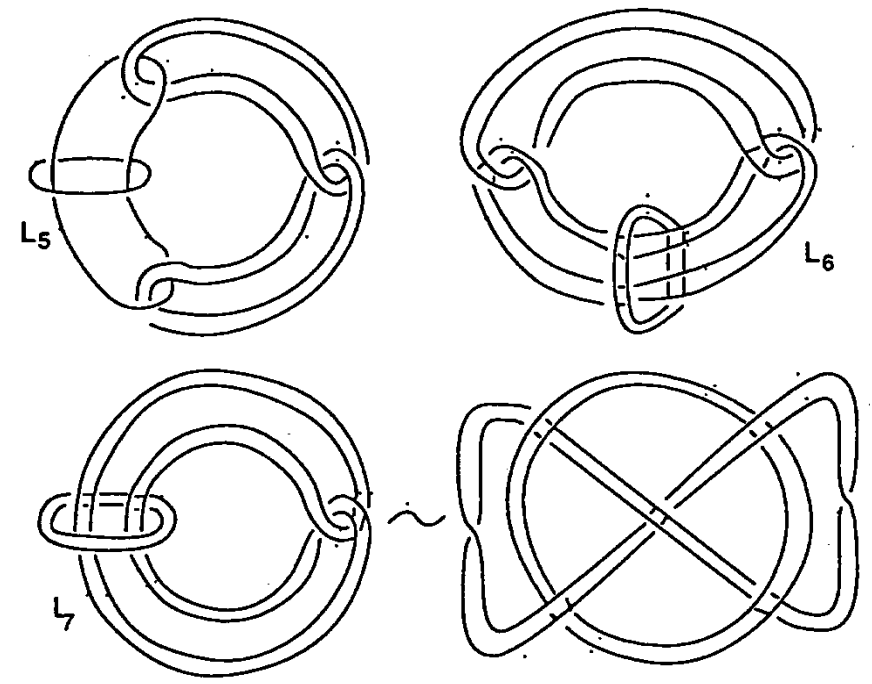

Figure 5.6.

The link $L_{7}$ is symmetric with respect to the $\pi$-rotation around the axis passing through $A$. Using $f_{2}^{\prime}$ we obtain $L_{8}$. The symmetry of $L_{2}$ produces $L_{9}$ by using an $f_{3}^{\prime}$. The symmetry of $L_{9}$ produces $L_{10}$. (Fig. 5.7.)
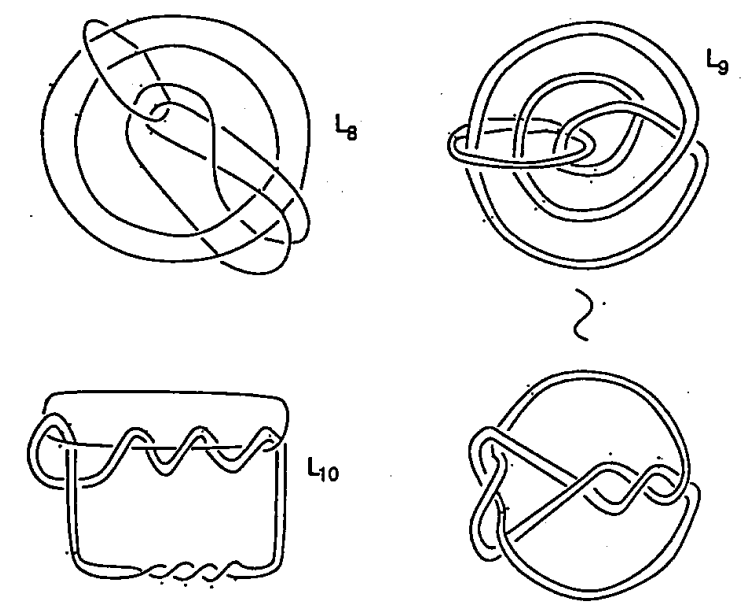

Figure 5.7.

We have proved the following theorem:

Theorem 5.1.4. The orbifolds $\left(L_{i}, 2\right),(i=1, \ldots, 10)$, are universal orbifolds. 
We now define a move $\mathcal{M}$ on the class of the orbifolds $(L, s)$.

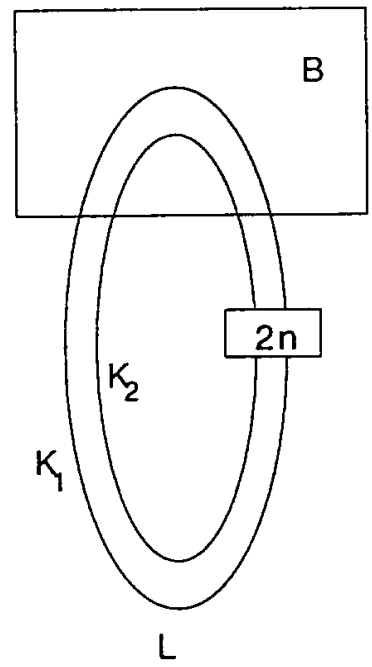

a)

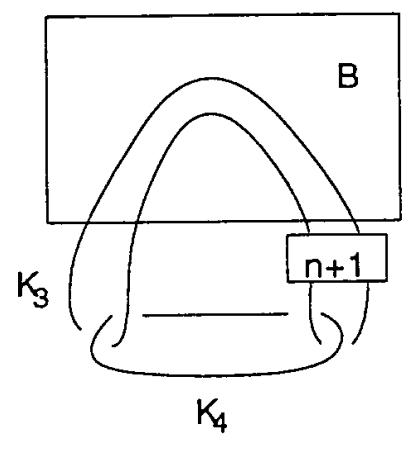

$\pi L$

b)

Figure 5.8.

Definition 5.1.5. Let $K_{1}, K_{2}$ be two unknotted components of a link $L$ linked as a $(2,2 n)$ toroidal link (see Fig. 5.8a)). Place the components of $L$ different from $K_{1}, K_{2}$ in the interior of the box $B$ of Fig. 5.8a). The link $\mathcal{M L}$ is obtained from $L$ by the replacement of the components $K_{1}, K_{2}$ by $K_{3}, K_{4}$ of Fig. $5.8 \mathrm{~b}$ ). Observe that $\mathcal{M} L$ is equal to $L$ in the interior of $B$.

Lemma 5.1.6. Let $(L, s)$ be a universal orbifold. Let $K_{1}$ and $K_{2}$ be two unknotted components of $L$ linked as a $(2,2 n)$ toroidal link. Then $(\mathcal{M} L, \lambda s)$ is also a universal orbifold, where $\lambda=1$ if $s$ is even, and $\lambda=2$ if $s$ is odd.

Proof. Figure 5.9 shows the 2-fold covering $p: S^{3} \longrightarrow S^{3}$ branched over $\mathcal{M} L$ such that $L \subset p^{-1}(\mathcal{M} L)$, as follows. Consider the projection of $L$ depicted in a), where the components of $L$ different from $K_{1}, K_{2}$ are placed in the interior of the box $B$. An isotropy out of $B$ changes a) in b). By the addition of new components in the interior of a new box $B^{\prime}$ we obtain a link symmetric with respect to the $\pi$-rotation around the axis $A$. c). The covering $p$ is the quotient of $S^{3}$ by this $\pi$-rotation. The component $K_{4}$ is $p(A)$.

Corollary 5.1.7. The orbifold $(L, 2)$, where $L$ is any link of Fig. 5.10, is a universal orbifold. 
a)

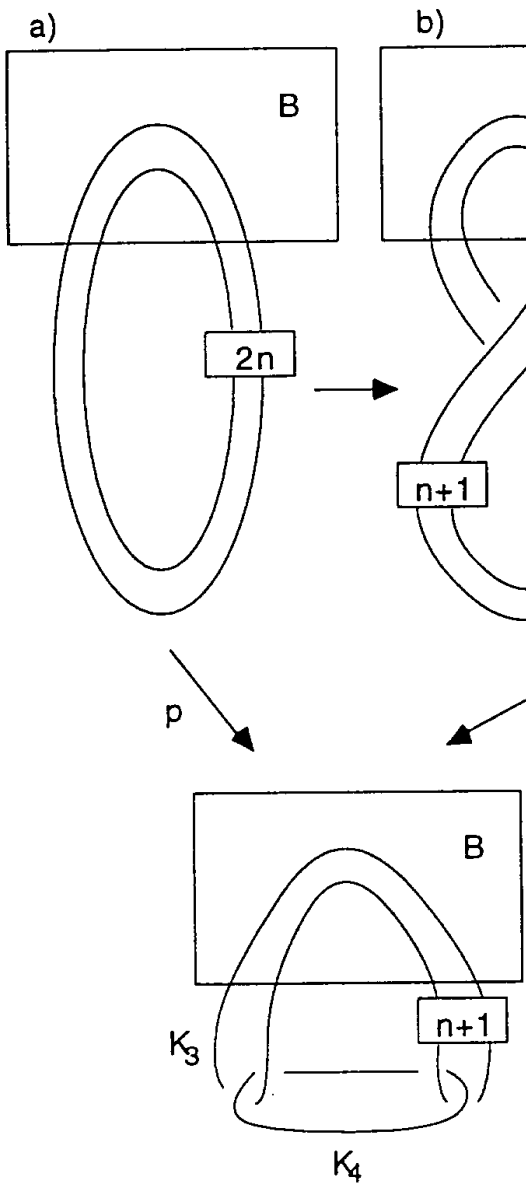

c)

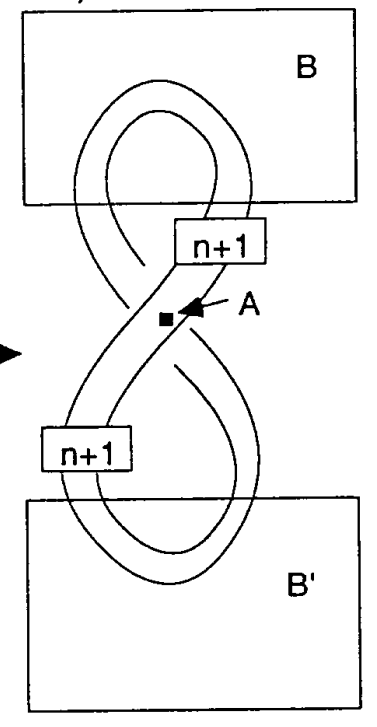

Figure 5.9. 

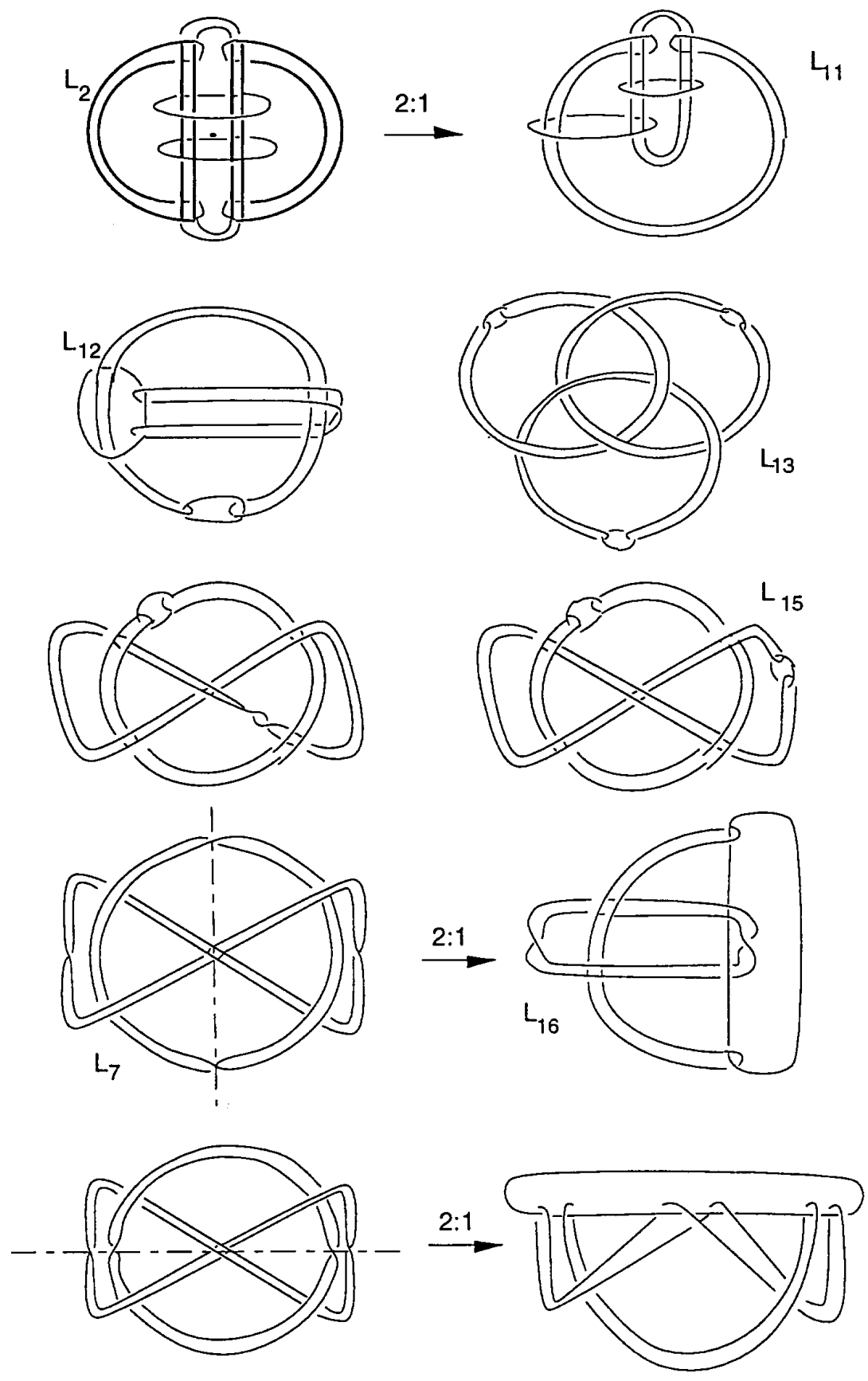

Figure 5.10. 
5.2. Flows in 3-manifolds. Let $p: M^{3} \longrightarrow N^{3}$ be a covering branched over a link $L \subset N^{3}$. Suppose that $L$ is a set of closed orbits of a flow $\varphi$ in $N^{3}$. Then there exists a flow $\psi$ in $M^{3}$ inducted by $\varphi$ and $p$. Moreover, if the flow $\varphi$ is hyperbolic in the exterior of $L, \psi$ is also hyperbolic in the exterior of $p^{-1}(L)$. Note that if the covering $p$ has a finite number of sheets, the preimage by $p$ of every closed orbit for the flow $\varphi$ is a finite set of closed orbits and the preimage of a dense orbit is a finite set of dense orbits. This implies that if $\varphi$ is a singular Anosov flow, $\psi$ is again a singular Anosov flow. The kind of the singular set of $\psi$ depends of the kind of the singular set of $\varphi$ and of the branched index of the points of $L$.

It is known [16] that not all 3-manifolds have Anosov flows. Using branched covering over $S^{3}$ we obtain some results about flows which can be defined in every 3 -manifold.

Theorem 5.2.1. Every closed and orientable 3-manifold $M^{3}$ has a transitive Anosov flow in the exterior of a link.

Proof. Let $M^{3}$ be a closed and orientable 3-manifold. There exists a finitefold covering $p: M^{3} \longrightarrow S^{3}$ branched over the figure eight knot $\left(4_{1}\right)$. [13]. The exterior of the knot $4_{1}$ is a torus bundle over $S^{1}$ with Anosov monodromy $\left(\begin{array}{ll}2 & 1 \\ 1 & 1\end{array}\right)$. Therefore $S^{3} \backslash 4_{1}$ has a transitive Anosov flow $\varphi$ that induces a transitive Anosov flow $\psi$ on $M^{3} \backslash p^{-1}\left(4_{1}\right)$.

Theorem 5.2.2. Every closed and orintable 3-manifold $M^{3}$ has a singular Anosov flow.

Proof. The closed braid $\widehat{\gamma}$ associated to the braid $\gamma=\Delta^{4} \bar{\sigma}_{1}^{4} \sigma_{2} \bar{\sigma}_{1}^{4} \sigma_{2}$ is the singular set of a $V$-Anosov flow $\varphi$ on $S^{3}$ (Theorem 4.2.1). (Fig. 5.11.) We will prove that $\widehat{\gamma}$ is also a universal link. The double cover of $S^{3}$ branched along the component $K$ of $\widehat{\gamma}$ is $S^{3}$ and the preimage of $\widehat{\gamma}$ is the link $L$ of Fig. 5.12. The sublink of $L$ made up of $K_{1}, K_{2}$ and $K_{3}$ is universal [12, Figure 11].

Then given a closed oriented 3-manifold $M^{3}$ there exists a finite fold covering map $p: M^{3} \longrightarrow S^{3}$ branched over the link $\widehat{\gamma}$. The flow inducted on $M^{3}$ by $\varphi$ and $p$ is a singular Anosov flow whose singular set $p^{-1}(\widehat{\gamma})$ is a finite set of closed orbits.

Theorem 5.2.3. Every closed and orientable 3-manifold $M^{3}$ has a singular Anosov flow, where the number of singular orbits which are not thorns is smaller or equal to 6.

Proof. The link $L$ of Fig. 5.13 is the singular set of the geodesic flow of the orbifold $D_{2222222222}$. 

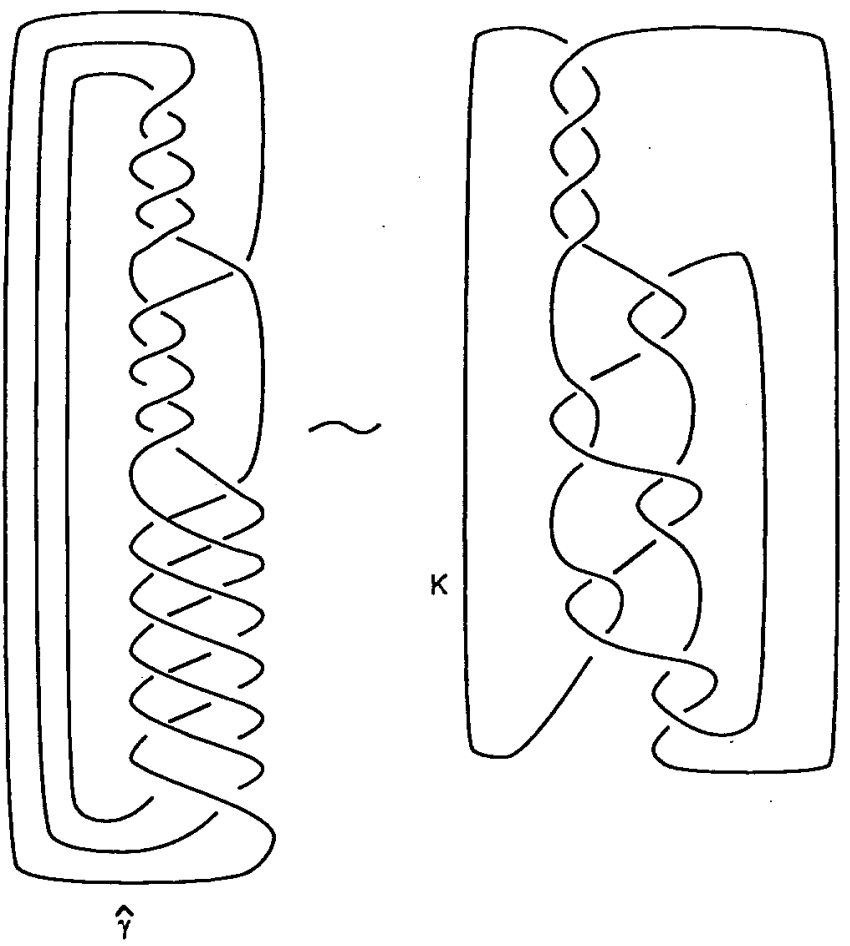

Figure 5.11.
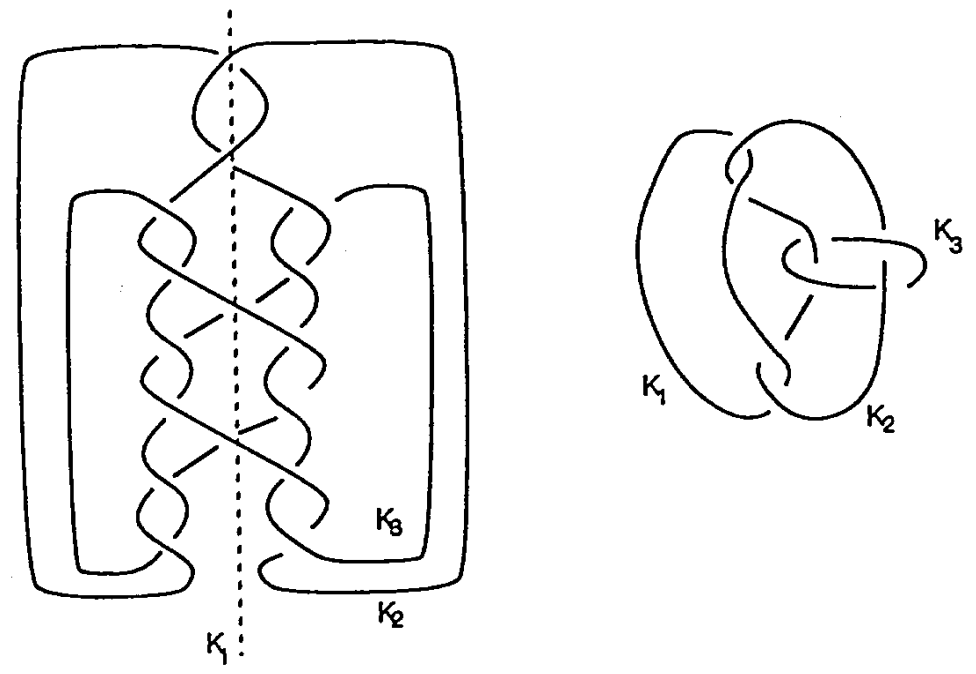

Figure 5.12.

$L$ is a universal link, such that $b\left(K_{i}\right)=2, i=3, \ldots, 10$. To prove that $L$ is a universal link, recall that given a closed oriented 3 -manifold $M^{3}$, there exists a simple 3 -fold covering map $p_{M}: M^{3} \longrightarrow S^{3}$ branched over a link $L_{M}$ of the following special form. $L_{M}$ can be placed in a neighbourhood of 
a torus. It has $m$ meridian components and $n$ longitude components and on each crossing of meridian components and longitude components is placed the pattern of Fig. 5.14. [12]. Observe that the link $L_{M}$ has a $\left(\mathbb{Z}_{m} \times \mathbb{Z}_{n}\right)$ symmetry. Then the composition $p^{\prime}=f_{n} \circ f_{m} \circ p: M^{3} \longrightarrow S^{3}$ is a covering branched over $L=f_{n} \circ f_{m}\left(L_{M}\right)$. The components $K_{1}$ and $K_{2}$ of $L$ are the image by $f_{n} \circ f_{m}$ of the $2 \pi / m$-rotation and $2 \pi / n$-rotation axis, respectively. Thus $b\left(K_{1}\right)=m, b\left(K_{2}\right)=n$ and $b\left(K_{i}\right)=2,(i=3, \ldots, 10)$. Note that $\left(f_{n} \circ f_{m} \circ p\right)^{-1}\left(K_{1} \cup K_{2}\right)$ has at most 6 components because $p$ is a 3 -fold covering. These are the only $k$-singular orbits, $k \geq 3$, in the inducted flow in $M^{3}$. The other singular orbits are thorns.

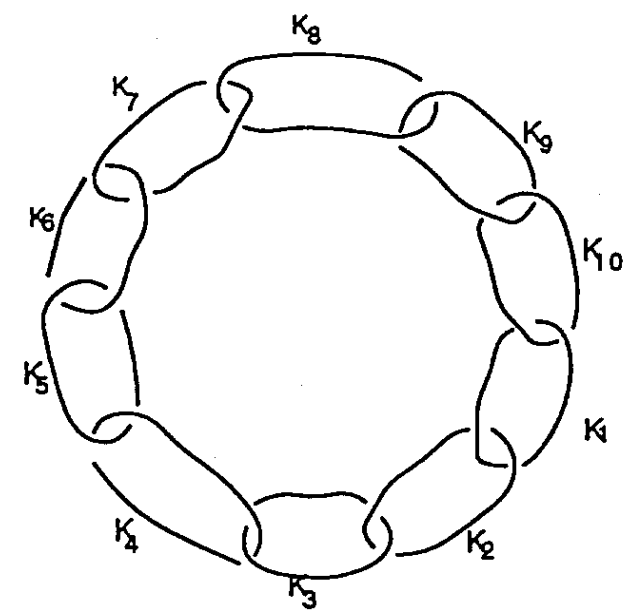

Figure 5.13.

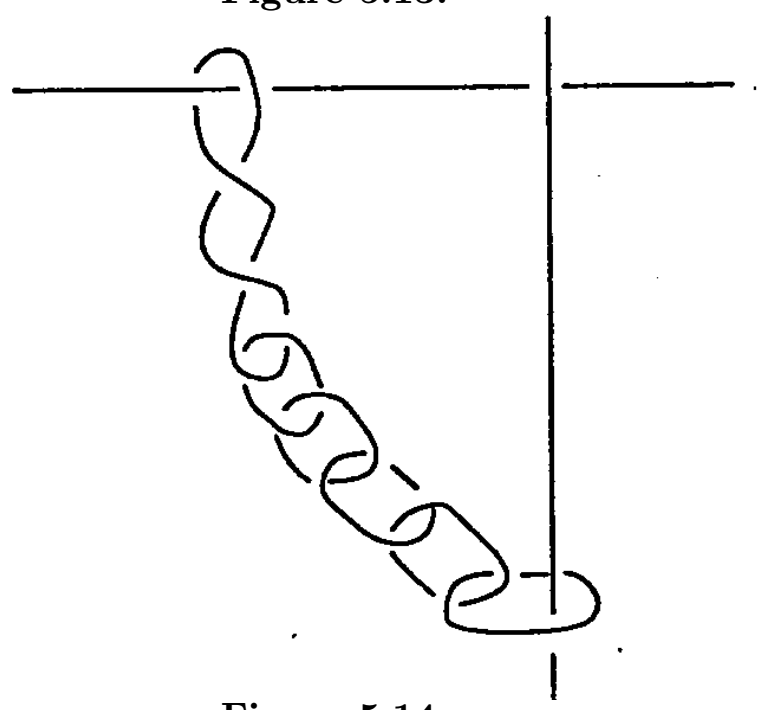

Figure 5.14. 
We end this paper with the following

Conjecture. Every closed and orientable 3-manifold $M^{3}$ has a $V$-Anosov flow.

\section{References}

[1] D.V. Anosov, Geodesic Flows on Closed Riemannian Manifolds with Negative Curvature, Proceeding of the Steklov Institute of Math., 90 (1967), English translations Amer. Math. Soc., (1969).

[2] J. Birman, Braids, links, and mapping class groups, Study 82, Princeton Univ. Press, 1976.

[3] F. Bonahon and L. Siebenmann, The classification of Seifert fibred 3-orbifolds, in Low Dimensional Topology. LMS. Lecture Note Series 95 (1985), 19-85.

[4] G. Burde and H. Zieschang, Knots, de Gruiter Studies in Mathematics, 5. Walter de Gruyter, Berlin, New York, 1985.

[5] J.P. Christy, Anosov Flows on three-Manifolds, Ph.D. Thesis. University of California, Berkeley, 1984.

[6] P. Eberlein, Geodesic flows on negatively curved manifolds I, Ann. of Math. (2), 95 (1972), 58-82.

[7] A. Fathi, F. Laudenbach and V. Poénaru, Travaux de Thurston sur les surfaces, Asterisque 66-67. Société Mathématique de France (1979).

[8] D. Fried, Transitive Anosov flows and pseudo-Anosov maps, Topology, 22 (1983), 299-303.

[9] E. Ghys, Flots d'Anosov dont les feuilletages stables sont differentiables, Ann. Sci. Ec. Norm. Super., IV. Ser., 20 (1987), 251-270.

[10] S. Goodman, Dehn surgery on Anosov flows, in Geometric Dynamics, SLN 1007, Springer, New York, (1983).

[11] J. Hempel, Construction of Orientable 3-manifold, in Topology of 3-manifold and related topics, Prentice-Hall (1962), 207-212.

[12] H. Hilden, M.T. Lozano and J.M. Montesinos, The Whitehead link, the Borromean rings and the knot $9_{46}$ are universal, Collect. Math., 34 (1983), 19-28.

[13] _ On knots that are universal, Topology, 24 (1985), 499-504.

[14] H. Hilden, M.T. Lozano, J.M. Montesinos and W. Whitten, On universal groups and three-manifold, Invent. Math., 87 (1987), 441-456.

[15] M. Handel and W. Thurston, Anosov flows on New three Manifolds, Invent. Math., 59 (1980), 95-103.

[16] G.A. Margulis, Y-Flows on three-dimensional manifolds, Appendix to Anosov-Sinai: "Some smooth ergodic systems". Uspekhi Math. Nauk, 22 (1967), 107-172; Russian Math. Surveys, 22 (1967), 103-168.

[17] J.M. Montesinos, Variedades de Seifert que son recubridores cíclicos ramificados de dos hojas, Bol. Soc. Mat. Mexicana, 18 (1973), 1-32.

[18] - Revêtement ramifies de noeuds, espaces fibrés de Seifert et scindements de Heegard, Publ. Orsay, (1980).

[19]_, Classical Tessellations and Three-Manifolds, Springer-Verlag (1987).

[20] Una nota a un teorema de Alexander, Revista Mat. Hisp.-Amer. (4), 32 
(1972), 167-187.

[21] L. Mosher, The classification of Pseudo-Anosovs, Low dimensional Topology and Klenian Groups. LMS LNS112. Cambridge University Press, 1986.

[22] J. Palis and W. de Melo, Geometric theory of Dinamical Systems, Springer-Verlag, New York (1982).

[23] D. Rolfsen, Knots and Links, Publish or Perish, Berkeley (1976).

[24] W. Thurston, On the geometry and dynamics of diffeomorphisms of surfaces, Bull. AMS, 19 (1988), 417-431.

[25] _ Universal links, preprint, 1982.

[26] R.F. Williams, The "DA" maps of Smale and structural stability, Proc. on Sym. in Pure Math., XIV (1970), 329-334.

Received July 25, 1993 and revised August 1, 1994. Both authors were supported by DGICYT, PB89-0105, PB92-236.

Departamento de Matemáticas

FACUlTad DE Ciencias

UNIVERSIDAD DE ZARAGOZA

ZARAGOZA 50009

SPAIN

E-mail address: tlozano@posta.unizar.es

AND

Departamento de Geometría y Topología

FACUltad de Matemáticas

UNIVERSIDAD COMPLUTENSE DE MADRID

MADRID 28040

SPAIN 